\title{
Application of different fertilizers to cabernet sauvignon vines: Effects on grape aroma accumulation
}

\author{
Rafia Salifu, Zhen Zhang, Faisal Eudes Sam, Jixin Li, Teng-Zhen Ma, Jing Wang, Shun-Yu Han \\ and Yu-Mei Jiang* \\ Gansu Key Laboratory of Viticulture and Enology, College of Food Science and Engineering, Gansu Agricultural \\ University, Lanzhou, Gansu Province, China
}

Received 22 July 2021

Accepted 26 November 2021

Pre-press 16 December 2021

Published 17 June 2022

\begin{abstract}
.
BACKGROUND: Vine nutrition affects the composition of grapes, but how it impacts the aroma of grapes is largely unknown. OBJECTIVE: This work aimed to investigate the effect of different fertilizers: chemical fertilizer (CF), sheep manure-based organic fertilizer $(\mathrm{OF}), 50 \%$ organic fertilizer $+50 \%$ chemical fertilizer $(\mathrm{O}+\mathrm{C}), 25 \%$ organic fertilizer $+25 \%$ chemical fertilizer $[1 / 2(\mathrm{O}+\mathrm{C})]$, and soil conditioner $(\mathrm{SC})$ on the aroma accumulation of Cabernet Sauvignon grapes.

METHODS: The treatments were applied and samples were collected in 2019 at weekly intervals from August 7 to September 22. The grapes' chemical characteristics and volatile compounds were analyzed.

RESULTS: The chemical results showed that the treatments had a positive effect on grapes, with a strong preference for the $1 / 2(\mathrm{O}+\mathrm{C})$ treatment. Grape aroma results showed that the concentrations of grape aromas in $\mathrm{O}+\mathrm{C}$-treated samples were lower than the other treatments. The OF treated samples had comparatively high $(24.8 \%)$ volatile concentrations during maturity compared to other treatments, including the control (15.9\%). Throughout development, samples treated with OF $(17.4 \%)$ and $\mathrm{CF}(15.7 \%)$ had higher volatile concentrations than samples treated with SC $(14.4 \%), 1 / 2(\mathrm{O}+\mathrm{C})(12.8 \%)$, and $\mathrm{O}+\mathrm{C}(12.4 \%)$. However, compared to SC-treated samples, samples treated with $1 / 2(\mathrm{O}+\mathrm{C})$ increased the accumulation of terpenes and esters. The principal component analysis (PCA) results showed that samples treated with OF were strongly correlated to carbonyls, terpenes, and esters during maturity.
\end{abstract}

CONCLUSION: The type and ratio of fertilizer used had a significant impact on the aroma profile of Cabernet Sauvignon grapes.

Keywords: Grape, organic fertilizer, chemical fertilizer, soil conditioner, developmental stage, aroma compound

\section{Introduction}

Aroma is an essential characteristic that varies significantly with grape maturity and ultimately determines the grape and wine quality. Aromatic components of wine are an important factor that reflects the nutritional information of the wine and influences consumer liking [1]. Depending on the origin of aroma compounds, they are classified either as primary, secondary, or tertiary aromas [2]. The varietal (primary) aromas are derived from grapes and vary depending on the cultivars and vineyard practices [3].

\footnotetext{
*Corresponding author: Yu-Mei Jiang, Gansu Key Laboratory of Viticulture and Enology, College of Food Science and Engineering, Gansu Agricultural University, Lanzhou 730070, Gansu Province, China. Tel.: +8613909466466; E-mail: jym316@126.com.
}

ISSN 1878-5093 @ 2022 - The authors. Published by IOS Press. This is an Open Access article distributed under the terms of the Creative Commons Attribution-NonCommercial License (CC BY-NC 4.0). 
Production of quality grapes depends on the relations between agronomic practices and the physiological responses of the vine $[3,4]$. Soil management and training systems affect the composition of the grapes and irrefutably the composition and quality of the wine [5,6]. Vine at different stages of development has different nutritional needs because they involve a complex sequence of biochemical changes [7,8]. Integrated nutrient management plays a vital role in enhancing the growth and productivity of crops, particularly the role of secondary and micronutrients is very significant [9]. Fertilizer application is an effective way to improve the yield and quality of grapes. However, according to Zhang et al. [7], the influence of fertilizers on grape berries depends on the type and dose applied. According to El-Badawy [9], an adequate supply of potassium increases berry color and polyphenol contents. The nitrogen content of vines affects the synthesis of volatile compounds, specifically higher alcohols, and ethyl esters [10]. Helwi et al. [11] found that soil nitrogen fertilization increased the concentrations of ethyl esters and alcohols (butanol, phenylmethanol, and (E)-Hexen-3-ol) whiles decreasing the concentrations of isoamyl alcohols and 2-phenylethanol. In a study of urea administration to Sauvignon Blanc and Merlot vines, Lasa et al. [12] found an increase in esters concentrations. However, when foliar nitrogen fertilizers were sprayed on Tempranillo vines, Garde-Cerdan et al. [13] detected an increase in 2-phenylethanol and a decrease in terpenoids concentrations. Furthermore, a vine's high nitrogen status causes grape maturation to delay, resulting in lower color and total soluble solids concentrations [5, 7]. As a result, adequate application of nutrients in the vineyard is required to avoid a decline in grape quality components.

Cabernet Sauvignon (Vitis vinifera L. cv.) originates from Bordeaux and is one of the world's most extensively cultivated varieties [10], especially in China [14]. Cabernet Sauvignon grapes are small, acidic, dark blue with thick skin, and very aromatic. Cabernet Sauvignon can acclimatize to a wide range of soil types. However, Cabernet Sauvignon thrives in poor and deep gravelly soils with warm temperatures [15]. The aroma description for this cultivar is fruity or floral and usually herbaceous due to its high levels of methoxypyrazines [16]. However, environmental changes and management practices affect the grape composition, particularly the aroma $[6,17$, 18].

Shoot growth, grape yield, and quality, and thus the quality of wine generated from the grapes, are all affected by the nutrient deficit in the vine. The application of fertilizers is the only remedy as it is known to alter the nutritional state of the vine. However, the impact of fertilization on the aromatic composition of the grape is an area of interest for most winegrowers. Ningxia grape base is known for producing high-quality wine grapes. However, due to the poor nature of the soil in this region, fertilization is an inevitable means of increasing productivity. Several studies on the effect of fertilization have been published [9, 13, 19-22], but most of these studies are focused on single fertilization, while few studies on the effect of organic fertilizer on grape quality are reported [19, 23, 24]. Moreover, long-term usage of single fertilizers depletes soil organic matter and causes the imbalance of soil nutrients, affecting grape aroma formation and reducing grape quality, consequently lowering the wine quality. Previous studies show that the application of organic fertilizers maintains soil productivity, increases soil nutrient availability, and promotes crop growth [19, 25, 26]. However, there are few reported studies on the comparison of these different fertilizers. Therefore, the impact of various fertilizers and their combinations on the aroma formation of Cabernet Sauvignon grapes was investigated to determine the optimal fertilization practices for the long-term development of high-quality wine grapes in the region.

\section{Materials and Methods}

\subsection{Study site}

The experimental study was conducted in the grape base of Yuquanying farm in Yinchuan in the Eastern foot of Helan Mountain (Ningxia, China) during the 2019 growing season on 16-year-old Cabernet Sauvignon vines grafted on 1103-Paulsen rootstocks in alkaline, calcareous soil. Vines were trained to a vertical trellis system in North-South orientation with $3.00 \mathrm{~m} \times 0.80 \mathrm{~m}$ spacing between rows and within a row. 


\subsection{Treatment, experimental design, and sampling}

Five different fertilizers, specifically, chemical fertilizer (CF), sheep-based organic fertilizer (OF), 50\% organic fertilizer $+50 \%$ chemical fertilizer $(\mathrm{O}+\mathrm{C}), 25 \%$ organic fertilizer $+25 \%$ chemical fertilizer $[1 / 2(\mathrm{O}+\mathrm{C})]$, and soil conditioner (SC) were applied evenly to different vine furrows $(20 \mathrm{~cm}$ wide and $40 \mathrm{~cm}$ deep) at three different stages. Fifty percent $(50 \%)$ of the total of each treatment was applied after the grape unearthed, $25 \%$ of the total of each treatment after the grape bloom, and $25 \%$ of the total of each treatment during grape veraison. Some grapevines were not treated with fertilizer and served as control (CK) samples. The applications were done in triplicates and conducted in a complete randomized block design with 6 treatments. All treatments were irrigated using a drip irrigation system and management practices such as pruning and pest control were consistent. The chemical properties of the soils (0-40 cm depth) (Supplementary Table S1) were determined as described by Wang et al. [24]. The length of the shoot was measured using a $5 \mathrm{~m}$ measuring tape. Grapes were manually sampled weekly during development from August 7 to September 22, 2019, and flash-frozen in liquid nitrogen before storage at $-80^{\circ} \mathrm{C}$.

\subsection{Fertilizers contents and amount applied}

Chemical Fertilizer (CF); Nitrogen (N): $360 \mathrm{~kg} / \mathrm{ha}$, Phosphorus (P): $180 \mathrm{~kg} / \mathrm{ha}$, and Potassium (K): $300 \mathrm{~kg} / \mathrm{ha}$.

Organic Fertilizer (OF); Completely fermented and matured sheep manure. 9 t/ha (Organic matter $>45 \%$ and $\left.\mathrm{N}+\mathrm{P}_{2} \mathrm{O}_{5}+\mathrm{K}_{2} \mathrm{O}>5 \%\right)$.

$50 \%(\mathrm{OF}+\mathrm{CF})-(\mathrm{O}+\mathrm{C}) ; 4.5 \mathrm{t} / \mathrm{ha}\left(\right.$ Organic matter $>45 \%$ and $\left.\mathrm{N}+\mathrm{P}_{2} \mathrm{O}_{5}+\mathrm{K}_{2} \mathrm{O}>5 \%\right)+(\mathrm{N}: 180 \mathrm{~kg} / \mathrm{ha}$, $\mathrm{P}$ : $90 \mathrm{~kg} / \mathrm{ha}$, and $\mathrm{K}: 150 \mathrm{~kg} / \mathrm{ha})$.

$25 \%(\mathrm{OF}+\mathrm{CF})-1 / 2(\mathrm{O}+\mathrm{C}) ; 2.25$ t/ha (Organic matter $>45 \%$ and $\left.\mathrm{N}+\mathrm{P}_{2} \mathrm{O}_{5}+\mathrm{K}_{2} \mathrm{O}>5 \%\right)+(\mathrm{N}: 90 \mathrm{~kg} / \mathrm{ha}, \mathrm{P}$ : $45 \mathrm{~kg} / \mathrm{ha}$, and $\mathrm{K}: 75 \mathrm{~kg} / \mathrm{ha})$.

Soil conditioner (SC); 3 t/ha, containing desulphurization waste $12 \%$, attapulgite $15 \%$, volcanic stone $11 \%$, biomass slag $13 \%$, bio-organic fertilizer $33 \%$, humic acid $11 \%$, polyacrylamide $3 \%$, and compound microbial bacteria (photosynthetic bacteria and phosphate-solubilizing bacteria) $2 \%$.

\subsection{Chemicals and reagents}

Reagents used were analytically pure, and the water used was purified with a Milli-Q purification system (Molecular, Chongqing, China). Sodium hydroxide $(\mathrm{NaOH})$ and sodium chloride $(\mathrm{NaCl})$ were both purchased from Sigma Aldrich (Shanghai, China). The internal standard (2-Octanol) was also from Sigma Aldrich (Shanghai, China).

\subsection{Determination of chemical parameters of cabernet sauvignon grapes obtained from different fertilizer treatments}

The chemical parameters ( $\mathrm{pH}$ and Total Soluble Solids) of the grapes were determined as reported by the OIV [27]. Approximately $100 \mathrm{~g}$ of frozen grapes were randomly selected and placed in a clean beaker. After thawing at room temperature, the berries were rinsed thoroughly and dried with filter paper. The berries were deseeded, pressed manually, and centrifuged at $8000 \mathrm{rpm}$ for 10 minutes to obtain a clear juice. The juice was then analyzed for TSS $\left({ }^{\circ}\right.$ Brix), using a PAL-1 pocket refractometer (Atago - A624124, Japan) and for $\mathrm{pH}$ using a pH meter (Inesa PHS-3E, China). Titratable acidity (TA) was determined according to Ju et al. [28] and expressed as g/L tartaric acid. The parameters were all determined in triplicates $(n=3)$. 


\subsection{Analysis of grape aroma compounds by HS-SPME-GC-MS}

The grape aroma compounds were determined using Headspace Solid Phase Microextraction Gas Chromatography-Mass Spectrometry (HS-SPME-GC-MS) as reported by other authors $[1,22]$ with slight modifications. Approximately $50 \mathrm{~g}$ of berries were deseeded, blended, and $5 \mathrm{~g}$ of the slurry weighed into a $20 \mathrm{ml}$ vial. A small magnetic stir bar, sodium chloride $(1 \mathrm{~g}, \mathrm{NaCl})$, and $10 \mu \mathrm{L}$ of internal standard (50 ppm, 2-Octanol) were added. The vial was then tightly capped and equilibrated in a water bath at $40^{\circ} \mathrm{C}$ for 30 minutes with agitation at $40 \mathrm{rpm}$. The volatile aroma in the headspace of the vial was absorbed using 50/30 $\mu \mathrm{m} \mathrm{DVB/CAR/PDMS} \mathrm{fiber.}$ The fiber was thermally desorbed in the injector port of GC-MS for 10 minutes after extraction. Samples were all determined in triplicates $(n=3)$.

Volatiles were analyzed using a gas chromatography-mass spectrometer system (TRACE 1310- ISQ, Thermo Fisher Scientific, San Jose, CA, USA) with a DB-WAX column $(60 \mathrm{~m} \times 2.5 \mathrm{~mm} \times 0.25 \mu \mathrm{m}$, Agilent Technology, Santa Clara, CA, USA). Helium (He) was the carrier gas at a flow rate of $1 \mathrm{ml} /$ minute. The injector temperature was $230^{\circ} \mathrm{C}$ and set for splitless injection. The GC temperature program started with an oven temperature of $50^{\circ} \mathrm{C}$ for 10 minutes, a temperature series of $3^{\circ} \mathrm{C}$ /minute to a final temperature of $180^{\circ} \mathrm{C}$, and a final time of 6 minutes. The ion source and transfer line temperature were set respectively at $250^{\circ} \mathrm{C}$ and $180^{\circ} \mathrm{C}$. The mass range was $50 \mathrm{~m} / \mathrm{z}$ to $350 \mathrm{~m} / \mathrm{z}$, operated in full scan mode with electron energy of $70 \mathrm{eV}$. The volatile compounds detected were identified by comparing their mass spectra with those in National Institute for Standards and Technology (NIST 14; search version 2.0) library. The retention indices calculated using C6-C21 n-alkane series (Supelco, Bellefonte, PA, USA) were compared with those reported in the literature or the NIST database (http://webbook.nist.gov/chemistry/cas-ser.html). Quantification analysis was carried out only for volatile compounds identified in at least two of the three replicates. Any other than this was viewed as artifacts and omitted from further analysis. The compounds were analyzed quantitatively by their relative response to the 2-octanol internal standard. Finally, concentrations of volatile compounds were obtained and expressed as $\mu \mathrm{g} / \mathrm{L}$.

\subsection{Statistical analysis}

All data and analysis were done in triplicates and reported as average means. The data were analyzed using One Way Analysis of variance (ANOVA) in IBM SPSS Statistical software program 26 for Windows (SPSS inc. Chicago, USA), and the mean was compared using Post hoc Tukey Test at $p<0.05$. The grape volatile compounds classes in the different samples were subjected to Principal component analysis (PCA) using OriginPro 2018 version (Northampton, MA, USA).

\section{Results and discussion}

\subsection{Chemical properties of cabernet sauvignon grapes obtained from different fertilizer treatments}

Table 1 shows the chemical properties of Cabernet Sauvignon grapes during development. Throughout the growth period, there were significant differences between treated and untreated samples for each parameter. The total soluble solids (TSS) increased throughout the study, with treated samples recording higher values than untreated samples on Harvest Date-9 (HD-9). The TSS value of $1 / 2(\mathrm{O}+\mathrm{C})$ was the highest among the treated samples. On HD-9, the untreated samples had lower $\mathrm{pH}$ and titratable acidity (TA) values than the treated samples. The lower values found in the untreated samples suggest the control vines were low in potassium (Supplementary Table S1). According to El-Badawy [9], a low potassium supply decreases $\mathrm{pH}$ and TSS content in the berries because potassium is responsible for the osmotic regulations and membrane transport in the vine. All of the 
Table 1

Effects of different fertilizer treatments on the chemical properties of Cabernet Sauvignon grapes on different harvest dates

\begin{tabular}{|c|c|c|c|c|c|c|c|c|c|c|}
\hline Sample & & HD-1 & HD-2 & HD-3 & HD-4 & HD-5 & HD-6 & HD-7 & HD-8 & HD-9 \\
\hline \multirow[t]{6}{*}{ TSS } & CK & $12.1 \pm 0.06^{\mathrm{a}}$ & $15.5 \pm 0.00^{c}$ & $16.6 \pm 0.06^{\mathrm{b}}$ & $17.3 \pm 0.00^{\mathrm{e}}$ & $19.4 \pm 0.06^{\mathrm{e}}$ & $18.4 \pm 0.00^{\mathrm{a}}$ & $19.9 \pm 0.00^{c}$ & $24.2 \pm 0.06^{\mathrm{e}}$ & $20.5 \pm 0.00^{\mathrm{a}}$ \\
\hline & $\mathrm{CF}$ & $12.4 \pm 0.00^{\mathrm{b}}$ & $15.7 \pm 0.06^{\mathrm{d}}$ & $18 \pm 0.00^{\mathrm{e}}$ & $17.1 \pm 0.06^{\mathrm{d}}$ & $18.3 \pm 0.00^{\mathrm{d}}$ & $19 \pm 0.06^{\mathrm{c}}$ & $20.7 \pm 0.00^{\mathrm{e}}$ & $22.7 \pm 0.06^{\mathrm{d}}$ & $21.3 \pm 0.00^{c}$ \\
\hline & $\mathrm{OF}$ & $14.33 \pm 0.06^{\mathrm{d}}$ & $15.37 \pm 0.06^{\mathrm{b}}$ & $16.00 \pm 0.00^{\mathrm{a}}$ & $16.8 \pm 0.00^{\mathrm{c}}$ & $17.1 \pm 0.00^{\mathrm{a}}$ & $21.53 \pm 0.06^{\mathrm{e}}$ & $20.7 \pm 0.00^{\mathrm{e}}$ & $21.43 \pm 0.06^{\mathrm{a}}$ & $22.4 \pm 0.00^{\mathrm{d}}$ \\
\hline & $\mathrm{O}+\mathrm{C}$ & $13.4 \pm 0.00^{\mathrm{c}}$ & $15.63 \pm 0.06^{\mathrm{d}}$ & $16.9 \pm 0.00^{\mathrm{c}}$ & $16.7 \pm 0.00^{\mathrm{b}}$ & $17.83 \pm 0.06^{\mathrm{b}}$ & $18.83 \pm 0.06^{\mathrm{b}}$ & $19.17 \pm 0.06^{\mathrm{b}}$ & $22.2 \pm 0.00^{\mathrm{b}}$ & $20.9 \pm 0.00^{\mathrm{b}}$ \\
\hline & $1 / 2(\mathrm{O}+\mathrm{C})$ & $15 \pm 0.00^{\mathrm{e}}$ & $14 \pm 0.00^{\mathrm{a}}$ & $18.2 \pm 0.00^{\mathrm{f}}$ & $18.6 \pm 0.00^{\mathrm{f}}$ & $18.03 \pm 0.06^{\mathrm{c}}$ & $19.2 \pm 0.00^{\mathrm{d}}$ & $20 \pm 0.00^{\mathrm{d}}$ & $22.43 \pm 0.06^{\mathrm{c}}$ & $23.8 \pm 0.00^{\mathrm{f}}$ \\
\hline & SC & $14.4 \pm 0.00^{\mathrm{d}}$ & $15.97 \pm 0.06^{\mathrm{e}}$ & $17.3 \pm 0.00^{\mathrm{d}}$ & $15.3 \pm 0.00^{\mathrm{a}}$ & $18.1 \pm 0.00^{c}$ & $18.5 \pm 0.00^{\mathrm{a}}$ & $17.4 \pm 0.00^{\mathrm{a}}$ & $22.2 \pm 0.00^{\mathrm{b}}$ & $22.87 \pm 0.06^{\mathrm{C}}$ \\
\hline \multirow[t]{6}{*}{ PH } & CK & $3.02 \pm 0.00^{\mathrm{b}}$ & $2.82 \pm 0.00^{\mathrm{b}}$ & $3.23 \pm 0.00^{\mathrm{a}}$ & $3.38 \pm 0.00^{\mathrm{a}}$ & $3.72 \pm 0.00^{f}$ & $4.00 \pm 0.01^{\mathrm{f}}$ & $3.68 \pm 0.01^{\mathrm{c}}$ & $3.87 \pm 0.01^{\mathrm{d}}$ & $3.71 \pm 0.00^{\mathrm{b}}$ \\
\hline & $\mathrm{CF}$ & $2.97 \pm 0.00^{\mathrm{a}}$ & $3.11 \pm 0.00^{\mathrm{e}}$ & $3.40 \pm 0.00^{\mathrm{c}}$ & $3.41 \pm 0.01^{\mathrm{b}}$ & $3.47 \pm 0.00^{\mathrm{b}}$ & $3.62 \pm 0.01^{\mathrm{b}}$ & $3.68 \pm 0.00^{\mathrm{c}}$ & $3.85 \pm 0.01^{\mathrm{c}}$ & $3.74 \pm 0.01^{\mathrm{c}}$ \\
\hline & $\mathrm{OF}$ & $3.22 \pm 0.00^{\mathrm{e}}$ & $2.97 \pm 0.01^{\mathrm{d}}$ & $3.36 \pm 0.00^{\mathrm{b}}$ & $3.41 \pm 0.00^{\mathrm{b}}$ & $3.60 \pm 0.01^{\mathrm{d}}$ & $3.81 \pm 0.01^{\mathrm{e}}$ & $3.74 \pm 0.01^{\mathrm{e}}$ & $3.91 \pm 0.01^{\mathrm{e}}$ & $3.74 \pm 0.00^{\mathrm{c}}$ \\
\hline & $\mathrm{O}+\mathrm{C}$ & $3.05 \pm 0.00^{c}$ & $3.25 \pm 0.01^{\mathrm{f}}$ & $3.35 \pm 0.00^{\mathrm{b}}$ & $3.47 \pm 0.01^{\mathrm{c}}$ & $3.40 \pm 0.00^{\mathrm{a}}$ & $3.58 \pm 0.00^{\mathrm{a}}$ & $3.60 \pm 0.00^{\mathrm{b}}$ & $3.55 \pm 0.01^{\mathrm{a}}$ & $3.66 \pm 0.00^{\mathrm{a}}$ \\
\hline & $1 / 2(\mathrm{O}+\mathrm{C})$ & $3.29 \pm 0.00^{\mathrm{f}}$ & $2.81 \pm 0.01^{\mathrm{a}}$ & $3.36 \pm 0.00^{\mathrm{b}}$ & $3.58 \pm 0.01^{\mathrm{d}}$ & $3.50 \pm 0.01^{\mathrm{c}}$ & $3.76 \pm 0.00^{\mathrm{d}}$ & $3.71 \pm 0.01^{\mathrm{d}}$ & $3.79 \pm 0.01^{\mathrm{b}}$ & $3.80 \pm 0.00^{\mathrm{d}}$ \\
\hline & $\mathrm{SC}$ & $3.20 \pm 0.01^{\mathrm{d}}$ & $2.87 \pm 0.01^{\mathrm{c}}$ & $3.39 \pm 0.00^{\mathrm{c}}$ & $3.49 \pm 0.01^{\mathrm{c}}$ & $3.70 \pm 0.01^{\mathrm{e}}$ & $3.73 \pm 0.00^{\mathrm{c}}$ & $3.52 \pm 0.01^{\mathrm{a}}$ & $3.96 \pm 0.01^{\mathrm{f}}$ & $3.93 \pm 0.01^{\mathrm{e}}$ \\
\hline \multirow[t]{6}{*}{ TA } & CK & $9.83 \pm 0.25^{\mathrm{b}}$ & $7.73 \pm 0.11^{\mathrm{bc}}$ & $7.00 \pm 0.18^{\mathrm{c}}$ & $6.54 \pm 0.10^{\mathrm{d}}$ & $3.93 \pm 0.20^{\mathrm{c}}$ & $3.66 \pm 0.06^{\mathrm{b}}$ & $4.50 \pm 0.06^{\mathrm{c}}$ & $3.19 \pm 0.06^{\mathrm{b}}$ & $2.82 \pm 0.20^{\mathrm{a}}$ \\
\hline & $\mathrm{CF}$ & $11.41 \pm 0.12^{\mathrm{d}}$ & $6.44 \pm 0.18^{\mathrm{a}}$ & $5.74 \pm 0.10^{\mathrm{a}}$ & $5.03 \pm 0.20^{\mathrm{ab}}$ & $3.69 \pm 0.07^{b}$ & $4.30 \pm 0.12^{\mathrm{c}}$ & $3.42 \pm 0.20^{\mathrm{a}}$ & $3.62 \pm 0.10^{\mathrm{c}}$ & $3.39 \pm 0.06^{\mathrm{c}}$ \\
\hline & OF & $9.80 \pm 0.15^{\mathrm{b}}$ & $7.65 \pm 0.35^{\mathrm{b}}$ & $5.97 \pm 0.23^{\mathrm{ab}}$ & $4.80 \pm 0.06^{\mathrm{ab}}$ & $3.26 \pm 0.06^{\mathrm{a}}$ & $2.82 \pm 0.00^{\mathrm{a}}$ & $3.56 \pm 0.06^{\mathrm{a}}$ & $2.82 \pm 0.20^{\mathrm{a}}$ & $2.95 \pm 0.12^{\mathrm{ab}}$ \\
\hline & $\mathrm{O}+\mathrm{C}$ & $9.53 \pm 0.15^{\mathrm{b}}$ & $6.58 \pm 0.12^{\mathrm{a}}$ & $6.31 \pm 0.15^{\mathrm{b}}$ & $5.14 \pm 0.10^{\mathrm{b}}$ & $4.73 \pm 0.10^{\mathrm{d}}$ & $3.56 \pm 0.06^{\mathrm{b}}$ & $4.03 \pm 0.20^{\mathrm{b}}$ & $3.15 \pm 0.12^{\mathrm{b}}$ & $3.56 \pm 0.12^{\mathrm{c}}$ \\
\hline & $1 / 2(\mathrm{O}+\mathrm{C})$ & $10.51 \pm 0.25^{\mathrm{c}}$ & $9.04 \pm 0.07^{\mathrm{d}}$ & $6.18 \pm 0.23^{\mathrm{ab}}$ & $4.73 \pm 0.10^{\mathrm{a}}$ & $3.22 \pm 0.10^{\mathrm{a}}$ & $3.73 \pm 0.17^{\mathrm{b}}$ & $3.73 \pm 0.10^{\mathrm{ab}}$ & $3.22 \pm 0.00^{\mathrm{b}}$ & $3.22 \pm 0.10^{\mathrm{bc}}$ \\
\hline & SC & $7.65 \pm 0.10^{\mathrm{a}}$ & $8.16 \pm 0.09^{c}$ & $5.75 \pm 0.08^{\mathrm{a}}$ & $6.07 \pm 0.15^{\mathrm{c}}$ & $5.10 \pm 0.06^{\mathrm{e}}$ & $4.20 \pm 0.06^{\mathrm{c}}$ & $3.62 \pm 0.00^{\mathrm{a}}$ & $3.15 \pm 0.12^{\mathrm{b}}$ & $3.56 \pm 0.12^{\mathrm{c}}$ \\
\hline
\end{tabular}

Data are mean $\pm \mathrm{SD}$. Values in the same column with different superscripts are significantly different $(P<0.05)$. $\mathrm{CK}=(\mathrm{Control}, \mathrm{Untreated} \mathrm{Grapes})$; $\mathrm{CF}=(\mathrm{Chemical} F \mathrm{Fertilizer})$; $\mathrm{OF}=($ Organic Fertilizer $) ; \mathrm{O}+\mathrm{C}=(50 \% \mathrm{CF}+50 \% \mathrm{OF}) ; 1 / 2(\mathrm{O}+\mathrm{C})=(25 \% \mathrm{CF}+25 \% \mathrm{OF})$ and $\mathrm{SC}=($ Soil Conditioner $) ; \mathrm{HD}=(\mathrm{Harvest} \mathrm{Date}) ; \mathrm{TSS}=(\mathrm{Total} \mathrm{Soluble} \mathrm{Solids})$ and $\mathrm{TA}=($ Titratable Acidity $)$. 
treatments had a positive impact on the average grape production and shoot growth, with notable differences between the treated vines and control plants (Supplementary Table S2).

\subsubsection{Total Soluble Solids (TSS)}

Except on HD-7, where OF and SC treated samples decreased slightly, the total soluble solids content of grape juice increased with each harvest date. Although the parameter changed very little during grape development, there was a clear difference between untreated and treated samples. This means that the treated vines' source and sink ratios were balanced, preventing competition among the sinks for carbohydrates, thereby improving grape quality and composition [21]. The samples had a TSS concentration ranging from $12.1^{\circ}$ Brix on HD- 1 to 23. $8^{\circ}$ Brix on HD-9. On HD-9, samples treated with $1 / 2(\mathrm{O}+\mathrm{C})$ had the highest TSS at $23.8^{\circ}$ Brix. The results are in line with what other researchers have found [29, 30]. However, according to Soubeyrand et al. [31], different fertilization treatments did not affect berry growth in their investigation. The work by Soubeyrand et al. [31] was only focused on nitrogen, whereas treatments in this study included a variety of other elements. For improved wine quality, according to van Schalkwyk and Archer [32], the sugar level of red wine grapes at harvest should range from $20.5^{\circ}$ Brix to $23.5^{\circ}$ Brix. As a result, when compared to control grapes, samples treated with $1 / 2(\mathrm{O}+\mathrm{C})$ may produce higher-quality wine.

\subsection{2. $P H$}

The $\mathrm{pH}$ values of the samples increased from HD-1 to HD-6. However, there were differences among samples from HD-6 to HD-9. On HD-9, the $\mathrm{pH}$ values of all samples decreased except for those treated with $\mathrm{O}+\mathrm{C}$ and $1 / 2(\mathrm{O}+\mathrm{C})$, which increased. The study found that samples treated with $1 / 2(\mathrm{O}+\mathrm{C})$ on HD-1 had a low $\mathrm{pH}$ of 2.81 , and $\mathrm{CK}$ samples on HD-6 had a high $\mathrm{pH}$ of 4.00. However, the final $\mathrm{pH}$ ranged from 3.66 in samples treated with $\mathrm{O}+\mathrm{C}$ to 3.93 in samples treated with $\mathrm{SC}$. The final $\mathrm{pH}$ of samples treated with $\mathrm{CF}$ and $\mathrm{OF}$ was both 3.74 , while the $\mathrm{pH}$ of $\mathrm{CK}$ samples was the lowest (3.71). The CK sample value measured is similar to those reported by Antalick et al. [29]. The results pattern shows that fertilization treatments slightly altered the $\mathrm{pH}$ content of Cabernet Sauvignon grapes. Other researchers came to similar conclusions [1, 18, 33, 34].

\subsubsection{Titratable Acidity (TA)}

Titratable acidity showed significant differences $(p<0.05)$ in the dynamics of Cabernet Sauvignon grapes development. From HD-1 to HD-9, the titratable acidity (TA) decreased considerably, and the pattern was consistent across all samples. The final TA ranged from 2.82 to 3.56 , with the lowest being $\mathrm{CK}$ and the highest being samples treated with both $\mathrm{O}+\mathrm{C}$ and $\mathrm{SC}$. These observations might be attributed to the chemical properties of the different fertilizer treatments since other factors such as grape type, season, climate, among others, were all the same. Antalick et al. [29] found a similar pattern. From fresh fruit to mature fruit, the titratable acidity values in their study decreased. The findings, however, contradict those of Deluc et al. [30] and Yue et al. [33], and the reasons for this could be due to differences in geographical areas, cultural practices, and treatments.

\subsection{Grape volatile composition}

Grape-oriented volatile compounds are primarily located in the skin and produced by a variety of metabolic mechanisms. Alcohols, esters, acids, terpenes, carbonyls, $\mathrm{C}_{13}$-norisoprenoids are among the volatile classes found in grapes [35]. Some of these compounds found in grapes are present in free odor-active forms, while others are present as bound, non-volatile precursors, serving as potential aroma reservoirs [36].

The concentrations of aromatic compounds detected in Cabernet Sauvignon grapes with various fertilization treatments are shown in Table 2-4. In general, the content and concentration of volatile compounds in Cabernet Sauvignon grapes were affected by the different fertilization treatments. Throughout grape development, there were significant differences $(p<0.05)$ between untreated and treated samples. The differences observed in the study could be attributed to the variations in their mineral composition because the amounts of macronutrients 
Table 2

Concentration of aromatic compounds characterizing pre-veraison stage of Cabernet Sauvignon grapes obtained from different fertilizer treatments

\begin{tabular}{|c|c|c|c|c|c|c|c|}
\hline \multirow[t]{2}{*}{ Compound } & \multirow{2}{*}{$\begin{array}{c}\mathrm{RI} \\
\text { (cal) }\end{array}$} & \multicolumn{6}{|c|}{ 2-Octanol Equivalent Concentration $(\mu \mathrm{g} / \mathrm{L})$} \\
\hline & & $\mathrm{CK}$ & $\mathrm{CF}$ & $\mathrm{OF}$ & $\mathrm{O}+\mathrm{C}$ & $1 / 2(\mathrm{O}+\mathrm{C})$ & $\mathrm{SC}$ \\
\hline \multicolumn{8}{|l|}{ Alcohols } \\
\hline 1-Pentanol & 1215 & $779.72 \pm 0.00^{\mathrm{c}}$ & $20.11 \pm 8.65^{\mathrm{b}}$ & $10.53 \pm 9.51^{\mathrm{ab}}$ & $5.45 \pm 2.60^{\mathrm{ab}}$ & $10.08 \pm 6.27^{\mathrm{ab}}$ & $2.27 \pm 2.94^{\mathrm{a}}$ \\
\hline (Z)-2-Penten-1-ol & 1327 & $47.62 \pm 3.26^{\mathrm{b}}$ & $2.58 \pm 0.81^{\mathrm{a}}$ & $1.83 \pm 1.16^{\mathrm{a}}$ & $1.54 \pm 0.32^{\mathrm{a}}$ & nd & nd \\
\hline 1-Hexanol & 1361 & $575 \pm 3.01^{\mathrm{a}}$ & $315.62 \pm 1.91^{\mathrm{a}}$ & $341.53 \pm 1.28^{\mathrm{a}}$ & $222 \pm 1.60^{\mathrm{a}}$ & $284 \pm 1.08^{\mathrm{a}}$ & $271.10 \pm 6.93^{\mathrm{a}}$ \\
\hline (Z)-3-Hexen-1-ol & 1391 & $305.55 \pm 2.09^{\mathrm{b}}$ & $17.50 \pm 4.00^{\mathrm{a}}$ & $9.27 \pm 6.56^{\mathrm{a}}$ & $16.67 \pm 8.10^{\mathrm{a}}$ & $20.93 \pm 5.10^{\mathrm{a}}$ & $23.28 \pm 6.83^{\mathrm{a}}$ \\
\hline (E)-2-Hexen-1-ol & 1413 & $626 \pm 1.14^{\mathrm{b}}$ & $237.88 \pm 5.97^{\mathrm{a}}$ & $238.86 \pm 1.51^{\mathrm{a}}$ & $163.51 \pm 5.25^{\mathrm{a}}$ & $253.13 \pm 2.25^{\mathrm{a}}$ & $237.61 \pm 3.32^{\mathrm{a}}$ \\
\hline 1-Octen-3-ol & 1455 & $89.04 \pm 3.97^{b}$ & $4.17 \pm 1.47^{\mathrm{a}}$ & $3.35 \pm 0.79^{\mathrm{a}}$ & $2.73 \pm 0.29^{\mathrm{a}}$ & $4.16 \pm 0.57^{\mathrm{a}}$ & $3.26 \pm 0.23^{\mathrm{a}}$ \\
\hline 2-Ethylhexanol & 1495 & $33.47 \pm 2.46^{\mathrm{b}}$ & $5.40 \pm 4.79^{\mathrm{ab}}$ & $3.82 \pm 2.01^{\mathrm{a}}$ & $5.14 \pm 4.99^{\mathrm{a}}$ & $4.20 \pm 5.17^{\mathrm{a}}$ & $3.79 \pm 2.28^{a}$ \\
\hline 1-Octanol & 1564 & $62.02 \pm 2.14^{\mathrm{b}}$ & $2.70 \pm 0.63^{\mathrm{a}}$ & $2.12 \pm 1.08^{\mathrm{a}}$ & $1.83 \pm 0.37^{\mathrm{a}}$ & $2.84 \pm 0.73^{\mathrm{a}}$ & $1.59 \pm 0.30^{\mathrm{a}}$ \\
\hline 1-Nonanol & 1666 & $26.87 \pm 2.79^{b}$ & $1.58 \pm 0.38^{\mathrm{ab}}$ & $1.81 \pm 1.28^{\mathrm{ab}}$ & $2.16 \pm 0.71^{\mathrm{ab}}$ & $2.21 \pm 1.07^{\mathrm{ab}}$ & $0.75 \pm 0.34^{\mathrm{a}}$ \\
\hline Phenylethyl Alcohol & 1922 & $325 \pm 2.74^{\mathrm{b}}$ & $5.39 \pm 1.29^{\mathrm{a}}$ & $4.05 \pm 2.85^{\mathrm{a}}$ & $3.08 \pm 0.40^{\mathrm{a}}$ & $5.55 \pm 3.79^{\mathrm{a}}$ & $6.54 \pm 5.85^{\mathrm{a}}$ \\
\hline Total Alcohols & & $2870.29 \pm 23.60^{c}$ & $612.93 \pm 29.9^{b}$ & $617.17 \pm 28.03^{b}$ & $424.11 \pm 24.63^{a}$ & $587.1 \pm 26.03^{b}$ & $550.19 \pm 29.02^{b}$ \\
\hline \multicolumn{8}{|l|}{ Carbonyls } \\
\hline Hexanal & 1084 & $369 \pm 1.30^{\mathrm{b}}$ & $147 \pm 1.22^{\mathrm{a}}$ & $153 \pm 1.02^{\mathrm{a}}$ & $210 \pm 3.94^{\mathrm{ab}}$ & $269 \pm 2.01^{\mathrm{ab}}$ & $228 \pm 2.60^{\mathrm{ab}}$ \\
\hline 3-Hexenal & 1147 & $42.46 \pm 3.32^{\mathrm{a}}$ & $4.33 \pm 2.78^{a}$ & $1.27 \pm 1.11^{\mathrm{a}}$ & $2.68 \pm 1.06^{\mathrm{a}}$ & $2.04 \pm 1.36^{\mathrm{a}}$ & $214.44 \pm 3.10^{\mathrm{a}}$ \\
\hline (E)-2-Hexenal & 1206 & $477 \pm 2.80^{\mathrm{a}}$ & $643 \pm 2.91^{\mathrm{a}}$ & $537 \pm 3.09^{\mathrm{a}}$ & $104 \pm 1.00^{\mathrm{a}}$ & $652 \pm 2.95^{\mathrm{a}}$ & $224 \pm 3.02^{\mathrm{a}}$ \\
\hline Octanal & 1294 & $34.21 \pm 2.44^{\mathrm{b}}$ & $0.49 \pm 0.28^{\mathrm{a}}$ & $0.56 \pm 0.52^{\mathrm{a}}$ & $0.98 \pm 0.58^{\mathrm{a}}$ & $2.43 \pm 1.92^{\mathrm{ab}}$ & $0.96 \pm 0.56^{\mathrm{a}}$ \\
\hline (E)-2-Heptenal & 1330 & $83.63 \pm 7.67^{\mathrm{b}}$ & $3.65 \pm 0.63^{\mathrm{a}}$ & $3.84 \pm 1.08^{\mathrm{a}}$ & $3.88 \pm 0.85^{\mathrm{a}}$ & nd & nd \\
\hline Nonanal & 1399 & $119.49 \pm 1.97^{\mathrm{b}}$ & $5.04 \pm 1.41^{\mathrm{a}}$ & $4.51 \pm 1.00^{\mathrm{a}}$ & $5.31 \pm 0.58^{\mathrm{a}}$ & $6.29 \pm 4.73^{\mathrm{a}}$ & $4.43 \pm 0.12^{\mathrm{a}}$ \\
\hline Oct-(2E)-enal & 1436 & $54.82 \pm 4.80^{\mathrm{b}}$ & $2.48 \pm 1.21^{\mathrm{a}}$ & $1.80 \pm 0.54^{\mathrm{a}}$ & $3.23 \pm 0.84^{\mathrm{a}}$ & $3.04 \pm 0.91^{\mathrm{a}}$ & $2.75 \pm 0.77^{\mathrm{a}}$ \\
\hline (E, E)-2, 4-Heptadienal & 1471 & $47.27 \pm 0.25^{\mathrm{b}}$ & $2.15 \pm 0.65^{\mathrm{a}}$ & $1.75 \pm 1.03^{\mathrm{a}}$ & $1.70 \pm 0.87^{\mathrm{a}}$ & $1.67 \pm 0.92^{\mathrm{a}}$ & $2.78 \pm 0.72^{\mathrm{ab}}$ \\
\hline Benzaldehyde & 1530 & $43.77 \pm 3.02^{\mathrm{b}}$ & $2.27 \pm 0.24^{\mathrm{a}}$ & $1.90 \pm 1.27^{\mathrm{a}}$ & $1.66 \pm 0.65^{\mathrm{a}}$ & $2.46 \pm 0.34^{\mathrm{a}}$ & $2.29 \pm 0.66^{\mathrm{a}}$ \\
\hline (E, Z)-2,6-Nonadienal & 1594 & $17.76 \pm 1.00^{\mathrm{b}}$ & $1.26 \pm 1.13^{\mathrm{ab}}$ & $0.54 \pm 0.45^{\mathrm{a}}$ & $0.91 \pm 0.19^{\mathrm{ab}}$ & $1.19 \pm 0.66^{\mathrm{ab}}$ & $0.69 \pm 0.71^{\mathrm{a}}$ \\
\hline Penten-3-one & 1025 & $5.33 \pm 0.00^{\mathrm{b}}$ & $0.20 \pm 0.03^{\mathrm{a}}$ & $0.15 \pm 0.01^{\mathrm{a}}$ & $0.27 \pm 0.15^{\mathrm{a}}$ & $0.39 \pm 0.42^{\mathrm{a}}$ & $0.18 \pm 0.03^{\mathrm{a}}$ \\
\hline 2-Octanone & 1290 & $12.71 \pm 0.00^{\mathrm{c}}$ & $0.51 \pm 0.11^{\mathrm{a}}$ & $0.71 \pm 0.35^{\mathrm{ab}}$ & nd & $1.95 \pm 1.17^{\mathrm{b}}$ & $0.64 \pm 0.32^{\mathrm{ab}}$ \\
\hline 6-Methylhept-5-en-2-one & 1343 & $108.26 \pm 1.41^{\mathrm{b}}$ & $4.60 \pm 2.40^{\mathrm{a}}$ & $3.31 \pm 1.32^{\mathrm{a}}$ & $4.54 \pm 4.13^{\mathrm{a}}$ & $7.64 \pm 4.93^{\mathrm{ab}}$ & $2.25 \pm 0.49^{\mathrm{a}}$ \\
\hline Total Carbonyls & & $1415.71 \pm 29.98^{d}$ & $816.98 \pm 15.00^{b c}$ & $710.34 \pm 12.79^{b}$ & $339.16 \pm 14.84^{a}$ & $950.1 \pm 22.32^{c}$ & $683.41 \pm 13.10^{b}$ \\
\hline \multicolumn{8}{|l|}{ Terpenes } \\
\hline Linalool & 1552 & $9.66 \pm 5.14^{b}$ & nd & $0.76 \pm 0.81^{\mathrm{a}}$ & $1.35 \pm 1.01^{\mathrm{a}}$ & $7.38 \pm 6.12^{b}$ & nd \\
\hline$\beta$-cyclocitral & 1632 & $24.57 \pm 1.36^{\mathrm{b}}$ & $1.98 \pm 0.56^{\mathrm{a}}$ & $1.57 \pm 0.43^{\mathrm{a}}$ & $1.92 \pm 1.26^{\mathrm{a}}$ & $1.93 \pm 0.71^{\mathrm{a}}$ & $1.95 \pm 0.74^{\mathrm{a}}$ \\
\hline Geraniol & 1855 & $33.17 \pm 0.77^{b}$ & $0.84 \pm 0.77^{\mathrm{a}}$ & $0.96 \pm 0.77^{\mathrm{a}}$ & $1.35 \pm 0.77^{\mathrm{a}}$ & $1.34 \pm 0.77^{\mathrm{a}}$ & $1.02 \pm 0.77^{\mathrm{a}}$ \\
\hline$\beta$-Ionone & 1952 & $20.84 \pm 0.07^{\mathrm{b}}$ & $0.49 \pm 0.07^{\mathrm{a}}$ & $0.63 \pm 0.07^{\mathrm{a}}$ & $0.35 \pm 0.07^{\mathrm{a}}$ & nd & $0.32 \pm 0.07^{\mathrm{a}}$ \\
\hline Total Terpenes & & $88.24 \pm 7.34^{c}$ & $3.31 \pm 1.40^{\mathrm{a}}$ & $3.92 \pm 2.08^{a}$ & $4.97 \pm 3.11^{\mathrm{a}}$ & $10.65 \pm 7.60^{b}$ & $3.29 \pm 1.58^{a}$ \\
\hline \multicolumn{8}{|l|}{ Esters } \\
\hline Hexyl acetate & 1278 & $3.85 \pm 0.00^{\mathrm{c}}$ & $0.70 \pm 0.03^{\mathrm{a}}$ & $1.21 \pm 0.91^{\mathrm{b}}$ & $0.71 \pm 0.01^{\mathrm{a}}$ & $0.90 \pm 0.06^{\mathrm{a}}$ & $0.81 \pm 0.09^{\mathrm{a}}$ \\
\hline (E)-2-Hexenyl acetate & 1340 & $8.19 \pm 0.55^{b}$ & $1.53 \pm 0.48^{\mathrm{a}}$ & $1.15 \pm 1.03^{\mathrm{a}}$ & $1.16 \pm 0.78^{\mathrm{a}}$ & $1.59 \pm 0.61^{\mathrm{a}}$ & $2.01 \pm 0.68^{\mathrm{a}}$ \\
\hline (3Z)-3-Hexen-1-yl acetate & 1323 & nd & nd & nd & $2.98 \pm 0.00$ & $7.55 \pm 0.00$ & nd \\
\hline Heptyl Formate & 1462 & $3.12 \pm 2.62^{b}$ & $2.01 \pm 0.77^{\mathrm{a}}$ & $1.90 \pm 0.70^{\mathrm{a}}$ & $1.67 \pm 0.33^{\mathrm{a}}$ & $1.94 \pm 0.67^{\mathrm{a}}$ & $1.48 \pm 0.19^{\mathrm{a}}$ \\
\hline Ethyl Octanoate & 1439 & $0.82 \pm 0.00^{\mathrm{ab}}$ & $0.64 \pm 0.05^{\mathrm{a}}$ & $0.67 \pm 0.00^{\mathrm{a}}$ & $1.02 \pm 0.64^{\mathrm{ab}}$ & $1.31 \pm 0.00^{\mathrm{b}}$ & $1.56 \pm 0.08^{\mathrm{b}}$ \\
\hline Ethyl hexanoate & 1238 & $4.57 \pm 0.00^{\mathrm{a}}$ & nd & nd & $1.41 \pm 0.11^{\mathrm{a}}$ & $0.48 \pm 0.00^{\mathrm{a}}$ & nd \\
\hline Ethyl hexadecanoate & 1441 & nd & nd & $0.60 \pm 0.00^{\mathrm{a}}$ & nd & $0.30 \pm 0.00^{\mathrm{a}}$ & nd \\
\hline
\end{tabular}


Table 2

(Continued)

\begin{tabular}{|c|c|c|c|c|c|c|c|}
\hline \multirow[t]{2}{*}{ Compound } & \multirow{2}{*}{$\begin{array}{c}\mathrm{RI} \\
(\mathrm{cal})\end{array}$} & \multicolumn{6}{|c|}{ 2-Octanol Equivalent Concentration $(\mu \mathrm{g} / \mathrm{L})$} \\
\hline & & $\mathrm{CK}$ & $\mathrm{CF}$ & $\mathrm{OF}$ & $\mathrm{O}+\mathrm{C}$ & $1 / 2(\mathrm{O}+\mathrm{C})$ & $\mathrm{SC}$ \\
\hline Ethyl benzoate & 1674 & nd & nd & nd & nd & nd & $0.35 \pm 0.00$ \\
\hline Ethyl decanoate & 1643 & nd & nd & nd & nd & $0.36 \pm 0.00$ & nd \\
\hline Methyl salicylate & 1788 & nd & nd & nd & $0.41 \pm 0.00$ & nd & nd \\
\hline (Z)-3-Hexenyl Butyrate & 1467 & nd & nd & nd & nd & $0.62 \pm 0.00$ & nd \\
\hline Total Esters & & $20.55 \pm 3.17^{c}$ & $4.88 \pm 1.33^{\mathrm{a}}$ & $5.53 \pm 2.64^{\mathrm{a}}$ & $9.36 \pm 1.87^{\mathrm{ab}}$ & $15.05 \pm 1.34^{b}$ & $6.21 \pm 1.04^{\mathrm{ab}}$ \\
\hline
\end{tabular}

Data are mean \pm SD. Values in a row with different superscripts are significantly different $(p<0.05)$ by the Tukey test. RI (cal), Retention indices calculated from the RT of series of straight-chain alkanes (C6-C20) using DB-WAX column. CK, samples without any treatment; $\mathrm{CF}$, samples treated with chemical fertilizer; OF, samples treated with organic fertilizer; $\mathrm{O}+\mathrm{C}$, samples treated with $50 \% \mathrm{CF}$ and $50 \% \mathrm{OF}$; $1 / 2(\mathrm{O}+\mathrm{C})$, samples treated with $25 \% \mathrm{CF}$ and $25 \% \mathrm{OF}$; SC, samples treated with soil conditioner; ND, not detected.

Table 3

Concentration of aromatic compounds characterizing veraison stage of Cabernet Sauvignon grapes obtained from different fertilizer treatments

\begin{tabular}{|c|c|c|c|c|c|c|c|}
\hline \multirow[t]{2}{*}{ Compound } & \multirow[t]{2}{*}{ RI (cal) } & \multicolumn{6}{|c|}{ 2-Octanol Equivalent Concentration $(\mu \mathrm{g} / \mathrm{L})$} \\
\hline & & CK & $\mathrm{CF}$ & OF & $\mathrm{O}+\mathrm{C}$ & $1 / 2(\mathrm{O}+\mathrm{C})$ & $\mathrm{SC}$ \\
\hline \multicolumn{8}{|l|}{ Alcohols } \\
\hline 1-Pentanol & 1216 & $13.62 \pm 2.09^{\mathrm{a}}$ & $305 \pm 1.95^{\mathrm{a}}$ & $8.05 \pm 0.91^{\mathrm{a}}$ & $8.06 \pm 0.42^{\mathrm{a}}$ & nd & $5.87 \pm 2.64^{\mathrm{a}}$ \\
\hline (Z)-2-Penten-1-ol & 1328 & $3.28 \pm 0.45^{\mathrm{a}}$ & $2.75 \pm 0.18^{\mathrm{a}}$ & $2.83 \pm 0.75^{\mathrm{a}}$ & $2.84 \pm 0.24^{\mathrm{a}}$ & $3.04 \pm 1.53^{\mathrm{a}}$ & $2.44 \pm 0.41^{\mathrm{a}}$ \\
\hline 1-Hexanol & 1361 & $316 \pm 4.92^{\mathrm{a}}$ & $348 \pm 9.48^{\mathrm{a}}$ & $503 \pm 2.18^{\mathrm{a}}$ & $244 \pm 3.05^{\mathrm{a}}$ & $400 \pm 1.21^{\mathrm{a}}$ & $254 \pm 8.46^{\mathrm{a}}$ \\
\hline (Z)-3-Hexen-1-ol & 1391 & $14.57 \pm 5.92^{\mathrm{a}}$ & $10.69 \pm 0.65^{\mathrm{a}}$ & $13.04 \pm 0.77^{\mathrm{a}}$ & $10.64 \pm 0.08^{\mathrm{a}}$ & $14.69 \pm 1.62^{\mathrm{a}}$ & $12.26 \pm 0.37^{\mathrm{a}}$ \\
\hline (E)-2-Hexen-1-ol & 1413 & $238 \pm 3.92^{\mathrm{a}}$ & $185 \pm 6.42^{\mathrm{a}}$ & $236 \pm 3.66^{\mathrm{a}}$ & $168 \pm 4.60^{\mathrm{a}}$ & $235 \pm 8.30^{\mathrm{a}}$ & $189 \pm 5.90^{\mathrm{a}}$ \\
\hline 1-Octen-3-ol & 1456 & $3.47 \pm 0.66^{\mathrm{a}}$ & $2.82 \pm 0.68^{\mathrm{a}}$ & $3.52 \pm 1.94^{\mathrm{a}}$ & $2.99 \pm 0.39^{\mathrm{a}}$ & $3.72 \pm 0.45^{\mathrm{a}}$ & $2.43 \pm 0.19^{\mathrm{a}}$ \\
\hline 2-Ethyl-1-hexanol & 1495 & $1.66 \pm 0.23^{\mathrm{a}}$ & $1.44 \pm 0.68^{\mathrm{a}}$ & $1.32 \pm 0.17^{\mathrm{a}}$ & $1.57 \pm 0.60^{\mathrm{a}}$ & $1.32 \pm 0.56^{\mathrm{a}}$ & $1.52 \pm 0.68^{\mathrm{a}}$ \\
\hline 1-Octanol & 1564 & $2.13 \pm 0.01^{\mathrm{a}}$ & $2.19 \pm 0.50^{\mathrm{a}}$ & $2.44 \pm 0.38^{\mathrm{a}}$ & $2.13 \pm 0.03^{\mathrm{a}}$ & $2.42 \pm 0.32^{\mathrm{a}}$ & $1.63 \pm 0.09^{\mathrm{a}}$ \\
\hline 1-Nonanol & 1668 & $1.12 \pm 0.27^{\mathrm{a}}$ & $0.94 \pm 0.64^{\mathrm{a}}$ & $1.17 \pm 0.17^{\mathrm{a}}$ & $0.82 \pm 0.31^{\mathrm{a}}$ & $1.41 \pm 0.36^{\mathrm{a}}$ & $0.73 \pm 0.12^{\mathrm{a}}$ \\
\hline Phenylethyl Alcohol & 1923 & $5.84 \pm 2.24^{\mathrm{a}}$ & $4.00 \pm 1.32^{\mathrm{a}}$ & $4.59 \pm 1.63^{\mathrm{a}}$ & $5.46 \pm 1.50^{\mathrm{a}}$ & $7.19 \pm 0.20^{\mathrm{a}}$ & $4.39 \pm 1.12^{\mathrm{a}}$ \\
\hline Nona-(2E,6Z)-dienol & 1775 & $1.43 \pm 0.41^{\mathrm{b}}$ & nd & $0.76 \pm 0.23^{\mathrm{ab}}$ & $0.34 \pm 0.14^{\mathrm{a}}$ & $0.98 \pm 0.55^{\mathrm{ab}}$ & $0.61 \pm 0.45^{\mathrm{ab}}$ \\
\hline Total Alcohols & & $601.12 \pm 21.12^{b}$ & $862.83 \pm 21.82^{c}$ & $776.72 \pm 12.79^{b c}$ & $446.85 \pm 11.36^{a}$ & $669.77 \pm 15.10^{b}$ & $474.88 \pm 20.43^{a}$ \\
\hline \multicolumn{8}{|l|}{ Carbonyls } \\
\hline Hexanal & 1087 & $201 \pm 6.78^{\mathrm{ab}}$ & $396 \pm 2.70^{\mathrm{b}}$ & $92.07 \pm 7.74^{\mathrm{a}}$ & $176 \pm 1.78^{\mathrm{a}}$ & $114 \pm 5.32^{\mathrm{a}}$ & $213 \pm 1.21^{\mathrm{b}}$ \\
\hline 3-Hexenal & 1146 & $3.64 \pm 1.15^{\mathrm{a}}$ & $2.67 \pm 0.06^{\mathrm{a}}$ & $2.03 \pm 0.62^{\mathrm{a}}$ & $3.48 \pm 1.37^{\mathrm{a}}$ & $1.34 \pm 0.40^{\mathrm{a}}$ & $2.84 \pm 2.17^{\mathrm{a}}$ \\
\hline (E)-2-Hexenal & 1207 & $157.46 \pm 0.77^{\mathrm{a}}$ & $138 \pm 1.55^{\mathrm{a}}$ & $405 \pm 2.42^{\mathrm{b}}$ & $321 \pm 3.84^{b}$ & nd & $613 \pm 2.13^{c}$ \\
\hline Octanal & 1293 & $0.94 \pm 1.38^{\mathrm{a}}$ & nd & nd & nd & nd & $0.36 \pm 0.17^{\mathrm{a}}$ \\
\hline Nonanal & 1399 & $4.69 \pm 2.99^{\mathrm{a}}$ & $2.93 \pm 0.98^{\mathrm{a}}$ & $3.56 \pm 1.57^{\mathrm{a}}$ & $4.09 \pm 0.29^{\mathrm{a}}$ & $3.37 \pm 1.51^{\mathrm{a}}$ & $3.10 \pm 0.53^{\mathrm{a}}$ \\
\hline (E, E)-2,4-Hexadienal & 1407 & $1.93 \pm 0.82^{\mathrm{a}}$ & $0.95 \pm 0.44^{\mathrm{a}}$ & nd & $1.33 \pm 0.54^{\mathrm{a}}$ & nd & nd \\
\hline Oct-(2E)-enal & 1437 & $2.35 \pm 0.83^{\mathrm{ab}}$ & $1.82 \pm 0.22^{\mathrm{ab}}$ & $1.68 \pm 0.61^{\mathrm{a}}$ & $3.69 \pm 0.14^{\mathrm{b}}$ & $2.35 \pm 0.47^{\mathrm{ab}}$ & $2.74 \pm 0.10^{\mathrm{ab}}$ \\
\hline (E, E)-2,4-Heptadienal & 1471 & $1.38 \pm 0.30^{\mathrm{a}}$ & $1.84 \pm 0.07^{\mathrm{a}}$ & $1.05 \pm 0.46^{\mathrm{a}}$ & $2.00 \pm 0.76^{\mathrm{a}}$ & $1.53 \pm 0.31^{\mathrm{a}}$ & $1.51 \pm 0.67^{\mathrm{a}}$ \\
\hline Benzaldehyde & 1531 & $190 \pm 0.85^{\mathrm{a}}$ & $1.96 \pm 0.39^{\mathrm{a}}$ & $1.45 \pm 0.50^{\mathrm{a}}$ & $1.81 \pm 0.44^{\mathrm{a}}$ & $1.68 \pm 0.12^{\mathrm{a}}$ & $1.51 \pm 0.57^{\mathrm{a}}$ \\
\hline Nona-(2E,6Z)-dienal & 1594 & $1.82 \pm 0.23^{\mathrm{a}}$ & $0.87 \pm 0.99^{\mathrm{a}}$ & $1.24 \pm 0.64^{\mathrm{a}}$ & $1.58 \pm 0.13^{\mathrm{a}}$ & $2.04 \pm 0.15^{\mathrm{a}}$ & $1.85 \pm 0.09^{\mathrm{a}}$ \\
\hline Penten-3-one & 1025 & $0.28 \pm 0.16^{\mathrm{a}}$ & $0.19 \pm 0.08^{\mathrm{a}}$ & nd & $0.35 \pm 0.20^{\mathrm{b}}$ & $0.15 \pm 0.02^{\mathrm{a}}$ & $0.26 \pm 0.03^{\mathrm{a}}$ \\
\hline
\end{tabular}


Table 3

(Continued)

\begin{tabular}{|c|c|c|c|c|c|c|c|}
\hline \multirow[t]{2}{*}{ Compound } & \multirow[t]{2}{*}{ RI (cal) } & \multicolumn{6}{|c|}{ 2-Octanol Equivalent Concentration $(\mu \mathrm{g} / \mathrm{L})$} \\
\hline & & $\mathrm{CK}$ & $\mathrm{CF}$ & $\mathrm{OF}$ & $\mathrm{O}+\mathrm{C}$ & $1 / 2(\mathrm{O}+\mathrm{C})$ & $\mathrm{SC}$ \\
\hline 2-Octanone & 1291 & $2.47 \pm 0.21^{\mathrm{a}}$ & $1.18 \pm 0.95^{\mathrm{a}}$ & $3.67 \pm 0.00^{\mathrm{a}}$ & $1.61 \pm 0.04^{\mathrm{a}}$ & $1.56 \pm 0.88^{\mathrm{a}}$ & $0.43 \pm 0.32^{\mathrm{a}}$ \\
\hline 6-Methylhept-5-en-2-one & 1344 & $3.93 \pm 0.71^{\mathrm{a}}$ & $3.66 \pm 1.73^{\mathrm{a}}$ & $5.04 \pm 2.54^{\mathrm{a}}$ & $3.67 \pm 0.43^{\mathrm{a}}$ & $4.05 \pm 1.13^{\mathrm{a}}$ & $1.92 \pm 0.84^{\mathrm{a}}$ \\
\hline Total Carbonyls & & $571.89 \pm 17.18^{b}$ & $552.07 \pm 10.16^{b}$ & $516.79 \pm 17.10^{b}$ & $520.61 \pm 9.96^{b}$ & $132.07 \pm 10.31^{\mathrm{a}}$ & $842.52 \pm 8.83^{c}$ \\
\hline \multicolumn{8}{|l|}{ Terpenes } \\
\hline Linalool & 1552 & $0.30 \pm 0.17^{\mathrm{a}}$ & $0.15 \pm 0.07^{\mathrm{a}}$ & $0.29 \pm 0.09^{\mathrm{a}}$ & $0.25 \pm 0.02^{\mathrm{a}}$ & $0.31 \pm 0.18^{\mathrm{a}}$ & $0.18 \pm 0.05^{\mathrm{a}}$ \\
\hline$\beta$-cyclocitral & 1633 & $0.96 \pm 0.33^{\mathrm{a}}$ & $1.13 \pm 0.21^{\mathrm{a}}$ & $0.93 \pm 0.23^{\mathrm{a}}$ & $0.91 \pm 0.08^{\mathrm{a}}$ & $1.05 \pm 0.15^{\mathrm{a}}$ & $1.01 \pm 0.07^{\mathrm{a}}$ \\
\hline Geraniol & 1855 & $1.70 \pm 0.07^{\mathrm{a}}$ & $2.61 \pm 0.07^{\mathrm{a}}$ & nd & $1.83 \pm 0.07^{\mathrm{a}}$ & $1.49 \pm 0.07^{\mathrm{a}}$ & nd \\
\hline Total Terpenes & & $2.96 \pm 0.57^{\mathrm{ab}}$ & $3.44 \pm 0.35^{b}$ & $1.22 \pm 0.32^{\mathrm{a}}$ & $2.99 \pm 0.17^{\mathrm{ab}}$ & $2.85 \pm 0.40^{\mathrm{ab}}$ & $1.19 \pm 0.12^{\mathrm{a}}$ \\
\hline \multicolumn{8}{|l|}{ Esters } \\
\hline Hexyl acetate & 1279 & $0.15 \pm 0.00^{\mathrm{a}}$ & $1.73 \pm 0.00^{\mathrm{a}}$ & nd & nd & nd & nd \\
\hline (E)-2-Hexenyl acetate & 1340 & nd & $0.94 \pm 0.19^{\mathrm{a}}$ & $0.23 \pm 0.11^{\mathrm{a}}$ & $0.25 \pm 0.12^{\mathrm{a}}$ & $0.38 \pm 0.08^{\mathrm{a}}$ & $0.45 \pm 0.31^{\mathrm{a}}$ \\
\hline Heptyl formate & 1462 & $2.46 \pm 0.00^{\mathrm{a}}$ & $1.40 \pm 0.07^{\mathrm{a}}$ & $2.13 \pm 0.26^{\mathrm{a}}$ & $1.80 \pm 0.11^{\mathrm{a}}$ & $2.23 \pm 0.42^{\mathrm{a}}$ & $1.48 \pm 0.19^{\mathrm{a}}$ \\
\hline Ethyl octanoate & 1442 & $0.21 \pm 0.00^{\mathrm{b}}$ & $0.74 \pm 0.00^{\mathrm{d}}$ & $0.16 \pm 0.03^{\mathrm{a}}$ & $0.63 \pm 0.00^{c}$ & nd & nd \\
\hline Ethyl decanoate & 1644 & $0.16 \pm 0.00$ & nd & nd & nd & $0.29 \pm 0.08$ & nd \\
\hline Ethyl hexadecanoate & 1442 & nd & nd & $0.93 \pm 0.00$ & nd & $0.71 \pm 0.21$ & nd \\
\hline Geranyl isovalerate & 1825 & $0.12 \pm 0.00$ & nd & nd & nd & nd & nd \\
\hline Total Esters & & $3.10 \pm 0.00^{\mathrm{a}}$ & $4.81 \pm 0.26^{\mathrm{a}}$ & $3.45 \pm 0.40^{\mathrm{a}}$ & $2.68 \pm 0.23^{a}$ & $3.61 \pm 0.79^{a}$ & $1.93 \pm 0.50^{\mathrm{a}}$ \\
\hline
\end{tabular}

Data are mean \pm SD. Values in a row with different superscripts are significantly different $(p<0.05)$ by the Tukey test. RI (cal), Retention indices calculated from the RT of series of straight-chain alkanes (C6-C20) using DB-WAX column. CK, samples without any treatment; $\mathrm{CF}$, samples treated with chemical fertilizer; OF, samples treated with organic fertilizer; O + C, samples treated with 50\% CF and 50\% OF; $1 / 2(\mathrm{O}+\mathrm{C})$, samples treated with $25 \% \mathrm{CF}$ and $25 \% \mathrm{OF}$; SC, samples treated with soil conditioner; ND, not detected.

Table 4

Concentration of aromatic compounds characterizing maturity stage of Cabernet Sauvignon grapes obtained from different fertilizer treatments

\begin{tabular}{|c|c|c|c|c|c|c|c|}
\hline \multirow[t]{2}{*}{ Compound } & \multirow[t]{2}{*}{ RI (cal) } & \multicolumn{6}{|c|}{ 2-Octanol Equivalent Concentration $(\mu \mathrm{g} / \mathrm{L})$} \\
\hline & & CK & $\mathrm{CF}$ & $\mathrm{OF}$ & $\mathrm{O}+\mathrm{C}$ & $1 / 2(\mathrm{O}+\mathrm{C})$ & $\mathrm{SC}$ \\
\hline \multicolumn{8}{|l|}{ Alcohols } \\
\hline 1-Pentanol & 1216 & $21.16 \pm 1.42^{\mathrm{a}}$ & $13.49 \pm 4.46^{\mathrm{a}}$ & $18.8 \pm 1.27^{\mathrm{b}}$ & $14.62 \pm 2.56^{\mathrm{a}}$ & $10.81 \pm 2.34^{\mathrm{a}}$ & $10.15 \pm 1.96^{\mathrm{a}}$ \\
\hline (Z)-2-Penten-1-ol & 1328 & $6.81 \pm 1.26^{\mathrm{a}}$ & $2.99 \pm 0.37^{\mathrm{a}}$ & $2.14 \pm 1.08^{\mathrm{a}}$ & $3.34 \pm 0.26^{\mathrm{a}}$ & $3.69 \pm 0.13^{\mathrm{a}}$ & $4.14 \pm 1.85^{\mathrm{a}}$ \\
\hline 1-Hexanol & 1361 & $408 \pm 5.98^{\mathrm{b}}$ & $235 \pm 2.18^{\mathrm{a}}$ & $238 \pm 4.96^{\mathrm{a}}$ & $385 \pm 4.79^{\mathrm{b}}$ & $195 \pm 7.08^{\mathrm{a}}$ & $342 \pm 6.83^{b}$ \\
\hline (Z)-3-Hexen-1-ol & 1391 & $9.17 \pm 2.08^{\mathrm{a}}$ & $6.02 \pm 1.04^{\mathrm{a}}$ & $22.14 \pm 2.14^{\mathrm{a}}$ & $8.82 \pm 1.12^{\mathrm{a}}$ & $2.62 \pm 0.48^{\mathrm{a}}$ & $6.08 \pm 1.33^{\mathrm{a}}$ \\
\hline (E)-2-Hexen-1-ol & 1413 & $229 \pm 5.71^{\mathrm{a}}$ & $134 \pm 6.56^{\mathrm{a}}$ & $79.00 \pm 4.92^{\mathrm{a}}$ & $188 \pm 1.22^{\mathrm{a}}$ & $118 \pm 5.71^{\mathrm{a}}$ & $140 \pm 1.86^{\mathrm{a}}$ \\
\hline 1-Octen-3-ol & 1456 & $4.94 \pm 1.77^{\mathrm{a}}$ & $5.68 \pm 1.44^{\mathrm{a}}$ & $18.89 \pm 2.04^{\mathrm{a}}$ & $4.92 \pm 0.07^{\mathrm{a}}$ & $4.73 \pm 1.13^{\mathrm{a}}$ & $5.79 \pm 1.32^{\mathrm{a}}$ \\
\hline 2-Ethylhexan-1-ol & 1495 & $1.36 \pm 0.22^{\mathrm{a}}$ & $0.87 \pm 0.55^{\mathrm{a}}$ & $6.09 \pm 1.34^{\mathrm{a}}$ & $1.37 \pm 0.19^{\mathrm{a}}$ & $0.97 \pm 0.40^{\mathrm{a}}$ & $1.24 \pm 0.30^{\mathrm{a}}$ \\
\hline 1-Octanol & 1564 & $3.00 \pm 0.79^{\mathrm{a}}$ & $1.63 \pm 0.18^{\mathrm{a}}$ & $13.12 \pm 1.81^{\mathrm{a}}$ & $2.33 \pm 0.13^{\mathrm{a}}$ & $1.95 \pm 0.44^{\mathrm{a}}$ & $2.60 \pm 0.49^{\mathrm{a}}$ \\
\hline 1-Nonanol & 1668 & $2.12 \pm 0.89^{\mathrm{a}}$ & $0.92 \pm 0.31^{\mathrm{a}}$ & $6.94 \pm 0.01^{\mathrm{a}}$ & $1.17 \pm 0.13^{\mathrm{a}}$ & $0.86 \pm 0.01^{\mathrm{a}}$ & $1.06 \pm 0.11^{\mathrm{a}}$ \\
\hline Phenylethyl Alcohol & 1922 & $8.56 \pm 2.78^{\mathrm{a}}$ & $5.57 \pm 1.97^{\mathrm{a}}$ & $4.26 \pm 5.29^{\mathrm{a}}$ & $5.80 \pm 0.20^{\mathrm{a}}$ & $5.99 \pm 0.53^{\mathrm{a}}$ & $4.91 \pm 0.92^{\mathrm{a}}$ \\
\hline (E, Z)-2, 6-Nonadien-1-ol & 1775 & $3.75 \pm 1.08^{\mathrm{a}}$ & $1.12 \pm 0.78^{\mathrm{a}}$ & $7.67 \pm 1.28^{\mathrm{a}}$ & $1.61 \pm 0.43^{\mathrm{a}}$ & nd & $1.69 \pm 0.11^{\mathrm{a}}$ \\
\hline Total Alcohols & & \multicolumn{6}{|c|}{ 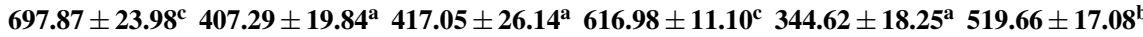 } \\
\hline
\end{tabular}


Table 4

(Continued)

\begin{tabular}{|c|c|c|c|c|c|c|c|}
\hline \multirow[t]{2}{*}{ Compound } & \multirow[t]{2}{*}{ RI (cal) } & \multicolumn{6}{|c|}{ 2-Octanol Equivalent Concentration $(\mu \mathrm{g} / \mathrm{L})$} \\
\hline & & CK & $\mathrm{CF}$ & OF & $\mathrm{O}+\mathrm{C}$ & $1 / 2(\mathrm{O}+\mathrm{C})$ & $\mathrm{SC}$ \\
\hline \multicolumn{8}{|l|}{ Carbonyls } \\
\hline Hexanal & 1087 & $132 \pm 1.76^{\mathrm{a}}$ & $277 \pm 2.54^{\mathrm{a}}$ & $572 \pm 8.14^{\mathrm{a}}$ & $137 \pm 3.19^{\mathrm{a}}$ & $72.61 \pm 3.22^{\mathrm{a}}$ & $74.32 \pm 6.86^{\mathrm{a}}$ \\
\hline 3-Hexenal & 1146 & $2.13 \pm 0.00^{\mathrm{a}}$ & $2.15 \pm 0.32^{\mathrm{a}}$ & $4.67 \pm 0.00^{\mathrm{a}}$ & $6.43 \pm 0.00^{\mathrm{a}}$ & $3.13 \pm 0.00^{\mathrm{a}}$ & $0.57 \pm 0.00^{\mathrm{a}}$ \\
\hline (E)-2-Hexenal & 1207 & $103.65 \pm 0.00^{\mathrm{a}}$ & $177 \pm 1.51^{\mathrm{ab}}$ & $257 \pm 2.43^{\mathrm{ab}}$ & $422 \pm 2.39^{b}$ & $183 \pm 3.81^{\mathrm{ab}}$ & $289 \pm 6.64^{\mathrm{ab}}$ \\
\hline Octanal & 1293 & nd & $0.39 \pm 0.12^{\mathrm{a}}$ & $1.01 \pm 3.19^{\mathrm{a}}$ & $0.29 \pm 0.10^{\mathrm{a}}$ & $0.61 \pm 0.12^{\mathrm{a}}$ & $0.32 \pm 0.00^{\mathrm{a}}$ \\
\hline Nonanal & 1399 & $3.76 \pm 1.09^{\mathrm{a}}$ & $4.32 \pm 0.70^{\mathrm{a}}$ & $21.96 \pm 0.03^{\mathrm{a}}$ & $3.40 \pm 0.62^{\mathrm{a}}$ & $3.29 \pm 0.66^{\mathrm{a}}$ & $4.75 \pm 1.95^{\mathrm{a}}$ \\
\hline Oct-(2E)-enal & 1436 & $1.13 \pm 0.36^{\mathrm{a}}$ & $1.29 \pm 0.40^{\mathrm{a}}$ & $6.01 \pm 1.97^{\mathrm{a}}$ & $1.82 \pm 0.46^{\mathrm{a}}$ & $1.67 \pm 0.51^{\mathrm{a}}$ & $1.72 \pm 0.37^{\mathrm{a}}$ \\
\hline (E, E)-2,4-Heptadienal & 1471 & $0.71 \pm 0.35^{\mathrm{a}}$ & $2.18 \pm 0.84^{\mathrm{a}}$ & $10.50 \pm 5.82^{\mathrm{b}}$ & $1.13 \pm 0.31^{\mathrm{a}}$ & $0.95 \pm 0.21^{\mathrm{a}}$ & $0.77 \pm 0.16^{\mathrm{a}}$ \\
\hline Benzaldehyde & 1530 & $1.52 \pm 0.36^{\mathrm{a}}$ & $2.67 \pm 0.15^{\mathrm{a}}$ & $21.49 \pm 3.72^{\mathrm{a}}$ & $1.58 \pm 0.41^{\mathrm{a}}$ & $1.58 \pm 0.37^{\mathrm{a}}$ & $1.43 \pm 0.47^{\mathrm{a}}$ \\
\hline Nona-(2E,6Z)-dienal & 1594 & $2.00 \pm 1.04^{\mathrm{a}}$ & $1.83 \pm 0.40^{\mathrm{a}}$ & $14.13 \pm 1.31^{\mathrm{a}}$ & $2.95 \pm 1.79^{\mathrm{a}}$ & $2.85 \pm 1.00^{\mathrm{a}}$ & $1.87 \pm 0.50^{\mathrm{a}}$ \\
\hline Penten-3-one & 1025 & nd & nd & $4.31 \pm 1.12^{\mathrm{a}}$ & nd & $0.27 \pm 0.12^{\mathrm{a}}$ & $0.16 \pm 0.06^{\mathrm{a}}$ \\
\hline 2-Octanone & 1290 & $6.38 \pm 2.29^{\mathrm{a}}$ & $1.86 \pm 0.55^{\mathrm{a}}$ & $9.08 \pm 1.50^{\mathrm{a}}$ & $2.77 \pm 2.84^{\mathrm{a}}$ & $1.07 \pm 0.34^{\mathrm{a}}$ & $4.30 \pm 4.52^{\mathrm{a}}$ \\
\hline 6-Methylhept-5-en-2-one & 1343 & $4.63 \pm 1.99^{\mathrm{a}}$ & $2.80 \pm 0.18^{\mathrm{a}}$ & $10.42 \pm 8.23^{\mathrm{a}}$ & $3.13 \pm 0.77^{\mathrm{a}}$ & $3.19 \pm 1.95^{\mathrm{a}}$ & $5.70 \pm 2.90^{\mathrm{a}}$ \\
\hline Total Carbonyls & & $257.91 \pm 9.24^{\mathrm{a}}$ & $473.49 \pm 7.71^{\mathrm{ab}}$ & $932.58 \pm 37.46^{\mathrm{c}}$ & $\mathbf{5 8 2 . 5 0} \pm \mathbf{1 2 . 8 8}^{\mathrm{b}}$ & $274.22 \pm 12.31^{\mathrm{a}}$ & $384.91 \pm 24.43^{a b}$ \\
\hline \multicolumn{8}{|l|}{ Terpenes } \\
\hline Linalool & 1552 & $0.24 \pm 0.01^{\mathrm{ab}}$ & $0.33 \pm 0.08^{b}$ & nd & $0.15 \pm 0.01^{\mathrm{a}}$ & $0.16 \pm 0.08^{\mathrm{a}}$ & nd \\
\hline$\beta$-cyclocitral & 1632 & $1.08 \pm 0.47^{\mathrm{a}}$ & $0.50 \pm 0.26^{\mathrm{a}}$ & $15.71 \pm 4.62^{\mathrm{b}}$ & $0.77 \pm 0.11^{\mathrm{a}}$ & $0.75 \pm 0.09^{\mathrm{a}}$ & $0.83 \pm 0.13^{\mathrm{a}}$ \\
\hline Geraniol & 1855 & $1.77 \pm 0.01^{\mathrm{b}}$ & nd & $14.23 \pm 0.01^{\mathrm{c}}$ & $0.26 \pm 0.03^{\mathrm{a}}$ & $1.46 \pm 0.07^{\mathrm{b}}$ & $0.19 \pm 0.01^{\mathrm{a}}$ \\
\hline Total Terpenes & & $3.09 \pm 0.49^{a}$ & $\mathbf{0 . 8 3} \pm 0.34^{\mathrm{a}}$ & $29.94 \pm 4.63^{b}$ & $1.18 \pm 0.15^{a}$ & $2.37 \pm 0.24^{\mathrm{a}}$ & $1.02 \pm 0.14^{a}$ \\
\hline \multicolumn{8}{|l|}{ Esters } \\
\hline Hexyl acetate & 1278 & nd & nd & nd & $0.07 \pm 0.00^{\mathrm{a}}$ & $0.90 \pm 1.37^{\mathrm{a}}$ & nd \\
\hline (E)-2-hexenyl acetate & 1340 & $0.55 \pm 0.33^{\mathrm{a}}$ & $0.13 \pm 0.00^{\mathrm{a}}$ & $39.98 \pm 6.79^{\mathrm{b}}$ & $0.19 \pm 0.06^{\mathrm{a}}$ & $0.30 \pm 0.14^{\mathrm{a}}$ & nd \\
\hline Heptyl formate & 1462 & $1.73 \pm 0.00^{\mathrm{a}}$ & $1.35 \pm 0.84^{\mathrm{a}}$ & $1.69 \pm 0.00^{\mathrm{a}}$ & $2.24 \pm 0.21^{\mathrm{a}}$ & $1.81 \pm 0.22^{\mathrm{a}}$ & $2.23 \pm 0.44^{\mathrm{a}}$ \\
\hline Ethyl octanoate & 1439 & nd & $0.44 \pm 0.00$ & nd & nd & nd & nd \\
\hline Ethyl hexanoate & 1238 & $11.06 \pm 0.00^{\mathrm{a}}$ & $0.49 \pm 0.58^{\mathrm{a}}$ & $48.20 \pm 6.06^{\mathrm{c}}$ & $9.67 \pm 0.00^{\mathrm{b}}$ & $7.40 \pm 6.32^{b}$ & $10.25 \pm 7.88^{b c}$ \\
\hline Ethyl (E)-2-hexenoate & 1351 & $1.11 \pm 0.00^{\mathrm{a}}$ & $0.27 \pm 0.13^{\mathrm{a}}$ & $58.14 \pm 7.96^{\mathrm{b}}$ & $0.93 \pm 0.00^{\mathrm{a}}$ & $1.29 \pm 1.02^{\mathrm{a}}$ & $1.41 \pm 1.11^{\mathrm{a}}$ \\
\hline Butyl Isobutyrate & 1879 & $0.61 \pm 0.00$ & nd & nd & nd & nd & nd \\
\hline Geranyl isovalerate & 1865 & $10.63 \pm 0.00$ & nd & nd & nd & nd & nd \\
\hline Total Esters & & $25.69 \pm \mathbf{0 . 3 3}^{b}$ & $2.68 \pm 1.55^{a}$ & $148.01 \pm 20.81^{c}$ & $13.10 \pm 0.27^{a b}$ & $11.70 \pm 9.07^{\mathrm{ab}}$ & $13.89 \pm 9.43^{\mathrm{ab}}$ \\
\hline
\end{tabular}

Data are mean \pm SD. Values in a row with different superscripts are significantly different $(p<0.05)$ by the Tukey test. RI (cal), Retention indices calculated from the RT of series of straight-chain alkanes (C6-C20) using DB-WAX column. CK, samples without any treatment; $\mathrm{CF}$, samples treated with chemical fertilizer; OF, samples treated with organic fertilizer; $\mathrm{O}+\mathrm{C}$, samples treated with $50 \% \mathrm{CF}$ and $50 \% \mathrm{OF}$; $1 / 2(\mathrm{O}+\mathrm{C})$, samples treated with $25 \% \mathrm{CF}$ and $25 \% \mathrm{OF}$; SC, samples treated with soil conditioner; ND, not detected.

and organic matter content (Supplementary Table S1) differed between the samples. The forty-two volatile compounds identified were grouped into four chemical categories; carbonyls, esters, terpenes, and alcohols.

\subsubsection{Carbonyls}

Carbonyls are straight-chain volatile compounds synthesized through $\beta$-oxidation and lipoxygenase (LOX) pathways by linoleic and linolenic acids metabolism [36]. The primary outcomes of the oxidative breakdown of these fatty acids are the $\mathrm{C}_{6}$ and $\mathrm{C}_{9}$ compounds. Other volatile compounds such as Hexyl acetate, (Z)-3-Hexen-1ol, (3Z)-Hexenyl acetate, etc., are formed when these primary products are further oxidized [36, 37]. The overall carbonyl concentrations decreased steeply from pre-veraison to maturity, with several $\mathrm{C}_{6}$-aldehydes (hexanal and 
E-2-hexenal) recording high concentrations. Hexanal and E-2-hexenal concentrations at pre-veraison accounted for $82 \%$ of total carbonyls, $90 \%$ at veraison, and $93 \%$ at maturity. The high concentrations found for these compounds imply the pathway for their synthesis was relatively active, which can be attributed to the impact of the treatments on the activities of enzymes in the LOX pathway. Previous reports [37, 38] state that lipoxygenases are involved in plant protection against biotic and abiotic stresses. The low N, P, and K contents in the control vines might have triggered the defense mechanisms of these enzymes in the pathway, which presumably led to the high accumulation of these $\mathrm{C}_{6}$-aldehydes. Moreover, during the volatile determination, the grapes were crushed, and according to Yuan [39], grape crushing enhances the formation of $\mathrm{C}_{6}$-compounds since it is a pre-fermentative step in winemaking. Due to the herbaceous odor impact of these compounds in wines, they receive much attention during wine production. Several fertilization investigations have revealed high levels of $\mathrm{C}_{6}$ compounds [12, 40, 41]. However, Yuan et al. [42] found a decrease in concentrations of (E)-2-hexenal and 1-hexanal in Pinot noir grapes supplemented with a low dose of nitrogen through soil application. Differences in grape variety and fertilizer dose could explain the observed variances because an adequate nitrogen content of a vine impacts the $\mathrm{C}_{6}$-compounds positively [5]. According to Yuan [39], low or soil nutrient deficiency limits the aroma potentials of grapes. The carbonyls concentrations at veraison and maturity were the highest in samples treated with SC (26.87\%) and OF (32.1\%), respectively and, could be attributed to the high organic matter content in these treatments. According to Coletta et al. [6], organic matter contains more beneficial microorganisms that regulate the availability of soil nutrients and obliquely enhance plant metabolism resulting in more synthesis of secondary metabolites. In a similar study, Yuan [39] found that the treated samples had higher carbonyls concentrations than the untreated samples; grape and wine aroma influenced by vine nutritional status, vigor, and crop levels in Oregon pinot noir. Alcohol dehydrogenase (ADH) enzymes convert carbonyls to their corresponding alcohols, which might explain the decreasing concentrations of carbonyls during development [43]. Conversely, the elevated concentrations found in samples treated with OF at maturity could be related to the enzyme specificity and the high organic matter content of the treatment [6,38,44]. Moreover, since other factors such as grape variety, management practices, and climate conditions were all the same, the concentration differences observed among samples may be attributed to the type and dose of fertilizer applied.

\subsubsection{Alcohols}

Alcohol compounds had the most abundant concentrations among the classes of compounds. However, carbonyls were the most abundant compounds in number. Although the overall alcohol concentrations decreased along with carbonyl concentrations during development, total alcohol concentrations were high (48.7\%) at maturity compared to other chemical classes. The results coincide with several researchers $[26,45]$ who stated that alcohols are characteristic compounds of the late stage of grape development. Although buildup of these compounds was substantial in samples treated with OF (10.9\%) and CF (10.8\%), total alcohol concentrations at pre-veraison were statistically the same in all treated samples. However, there was a significant difference between the treatments and the control group, with the control group showing 50.7\% of total alcohol concentrations. This finding could be related to the defense mechanism of enzymes in the biosynthetic pathway against the stress of low macronutrients (N, P, and K) in the control soil samples [37, 38]. The control samples showed no significant variation in total alcohol concentrations during the latter two stages of growth. Similarly, a study by Kalua and Boss [36] found no significant differences in alcohol concentrations between veraison and maturity stages. The total alcohol levels of $\mathrm{CF}, \mathrm{OF}$, and $1 / 2(\mathrm{O}+\mathrm{C})$ treated samples, on the other hand, decreased from veraison to maturity, with $\mathrm{CF}$ having the maximum concentration $(22.52 \%)$ during veraison. CK (23.24\%), $\mathrm{O}+\mathrm{C}(20.54 \%)$, and SC $(17.3 \%)$ had the highest total alcohol concentrations at maturity. Samples treated with CF $(13.56 \%)$ and OF $(13.88 \%)$ had statistically similar results, whereas samples treated with $1 / 2(\mathrm{O}+\mathrm{C})$ had the lowest results $(11.47 \%)$. The low concentrations of alcohols observed in the treated samples compared to control could be due to the diverse mechanisms of action of the different fertilizers applied. Alcohols are synthesized by various substrates, among which is the metabolism of amino acids. However, various cultural practices influence the compositions of amino acids in grapes [41]. With proper fertilization, the nitrogenous components of grapes 
rise, boosting the precursors for alcohol production. However, based on the results of the treated samples, it can be assumed that $\mathrm{CF}$ and $\mathrm{OF}$ negatively affected the nitrogenous components, resulting in decreased levels of alcohols while $\mathrm{O}+\mathrm{C}$ treatment with a modest amount of chemical and organic fertilizers increased the alcohol levels. These findings are consistent with earlier research on foliar application of urea to Sauvignon Blanc and Merlot vines [12]. The most abundant alcohols observed in this study were 1-hexanol and (E)-2-hexenol as also reported by Gutiérrez-Gamboa et al. (10]. However, Gutiérrez-Gamboa et al. [46], in a different study, found that 1-octen-3-ol was the most abundant alcohol. The differences observed in the latter study compared to the former could be explained by the different grape varieties studied. Other alcohols such as 1-octen-3-ol, 1-octanol, and 1-nonanol at maturity accounted for $42 \%, 53.3 \%$, and $53.1 \%$ concentrations respectively in OF treated samples, compared to the low concentrations observed in the other treated samples, including control. Since the influence of various treatments affects substrate concentration and enzyme specificity and activity differently [30, 47], the alcohol accumulation differences observed could be attributed to the compositions of the different fertilizers and dosages applied.

\subsubsection{Esters}

The fertilizer treatments affected the identified esters and their concentrations in various ways. The treatments increased the levels of some esters, delayed the accumulation of some, and inhibited the synthesis of others. Alcohols and aldehydes derived from grapes serve as precursors for esters biosynthesis [37, 48], particularly the $\mathrm{C}_{6}$-compounds (E)-2-hexenal, hexanal, (E)-2-hexenol, and hexanol. The oxidation of these compounds leads to the formation of Hexyl acetate, (E)-2-Hexenyl acetate, (3Z)-Hexenyl acetate, among other volatile compounds $[36,49]$. However, despite the high levels of $\mathrm{C}_{6}$ compounds found in the study, the content of esters reduced significantly, similar to the report by Vilanova et al. [50] on the chemical compositions of Albarino grapes after fertigation. The inhibition or low concentrations of esters could be due to the effects of fertilizers on the activities of related enzymes in the esters' biosynthetic pathways. According to previous reports [51,52], the synthesis of volatiles is influenced by enzyme activity and specificity as well as substrate concentration, implying that the trend in esters accumulations observed in this study could be related to the enzyme activity and specificity since the substrate concentrations $\left(\mathrm{C}_{6}\right.$ compounds) were abundant. For instance, Kalua and Boss [36] observed that the substrate (hexanol) was present, but hexyl acetate did not form due to the specificity of the related enzyme. Moreover, the low ester concentrations in our study suggest the activity of alcohol acetyltransferase (AAT) during maturation was lesser, which explains the trivial impact of esters in ripe grapes. Among the 14 esters identified, only hexyl acetate, (E)-2-hexenyl acetate, heptyl formate, and ethyl octanoate were found throughout development. The concentrations of these fatty acids and alcohols derived esters decreased in all samples, except for samples treated with $\mathrm{O}+\mathrm{C}$ and $\mathrm{SC}$, which recorded increasing concentrations of heptyl formate ranging from (13.8-20.3\%) and (12.2-20.2\%), respectively. Ethyl hexadecanoate and ethyl decanoate were identified only during the pre-veraison and veraison stages in some treated samples. The former was identified in samples treated with $\mathrm{OF}$ and $1 / 2(\mathrm{O}+\mathrm{C})$ with minor concentration differences, whereas the latter was discovered only in samples treated with $1 / 2(\mathrm{O}+\mathrm{C})$. Conversely, geranyl isovalerate was found only in control samples from veraison to maturity with increased concentrations $(0.12-10.63 \mu \mathrm{g} / \mathrm{L})$. Since the only factor difference between the samples was the treatment, the variance observed indicates that the fertilizers influenced the accumulation of geranyl isovalerate in the treated samples, probably due to the dosages applied. During development, the total esters concentrations among samples fluctuated with control (33.4\%) dominating the pre-veraison stage, $\mathrm{CF}$ samples $(24.6 \%)$ at veraison, and OF samples $(68.8 \%)$ at maturity. Except for the mature stage, $1 / 2(\mathrm{O}+\mathrm{C})$ samples were the second-highest in every case. Although accumulation of individual esters in the samples varied, the total esters concentrations among samples observed no significant difference during veraison. Kalua and Boss [36] reported similar findings in their study and postulated that esters bind as non-volatile conjugates during growth. Throughout development, samples treated with $\mathrm{O}+\mathrm{C}, 1 / 2(\mathrm{O}+\mathrm{C})$, and $\mathrm{SC}$ observed no differences in total esters concentrations. According to other investigations $[36,49]$, the quantity and quality of esters decrease as berries advance in growth. The findings substantiate the results in this study because the total number of 
esters during pre-veraison reduced as the berries matured. Moreover, the concentrations of grape-derived esters are lower than the esters concentrations in wines because esters are the metabolic products of yeast during winemaking [53, 54], which impacts the fermented product with fruity and floral aromas. Although the overall esters concentrations in samples treated with $1 / 2(\mathrm{O}+\mathrm{C})$ were statistically similar to $\mathrm{CK}, \mathrm{O}+\mathrm{C}$, and SC samples, it increased the accumulation of more esters than the other treatments.

\subsubsection{Terpenoids}

Terpenoids are synthesized through Mevalonate (MVA) and Methylerythritol phosphate (MEP) pathways and are related to the secondary metabolism of plants [37]. The few terpenoids detected in this study decreased significantly in concentration in all samples except samples treated with OF, which increased the concentrations of $\beta$-cyclocitral (80\%) and Geraniol (79.5\%) at the maturity stage. Linalool concentrations during development only increased in samples treated with $\mathrm{CF}$, reaching a final concentration of $37.5 \%$ compared to $27.3 \%$ in control. The type of fertilizer used may have contributed to the elevated concentrations of these terpenes in the treated samples (CF and $\mathrm{OF}$ ). The mechanism of action of fertilizers on substrate concentration, enzyme activity, and enzyme specificity differ with the synthesis of different terpenes $[52,55]$. Terpenoids found in $\mathrm{CK}, \mathrm{O}+\mathrm{C}$, and $1 / 2(\mathrm{O}+\mathrm{C})$ samples decreased in concentration throughout development. The results obtained are consistent with the findings of $\mathrm{Wu}$ et al. [1]. They observed that the buildup of monoterpenes at the fruit set stage declined, and accumulation only resumed at pre-veraison. A study on the evolution of volatile compounds during the development of Cabernet Sauvignon grapes also reported decreased concentrations of terpenes [36]. According to previous works $[22,36,49]$, the concentration of terpenes in neutral and non-Muscat aromatic grapes decreases significantly during grape ripening. Researchers relating to this discovery made some assumptions since there have been no investigations on the continued metabolism of terpenes into other volatile compounds. According to Kalua and Boss [36], terpenes during veraison are converted into bound glycosylated forms, whereas others speculate that the pathways to terpenes synthesis could have been suppressed [7, 56]. Moreover, the continual reduction in concentration and number of terpenes may be due to their role in plant processes like photosynthesis, membrane structure, and growth regulation [57], or their high vapor pressure, which allows their release into the atmosphere [37]. According to the findings in this study, Cabernet Sauvignon grapes are within the class of neutral aromatic varieties, implying that terpenes contribute little to the aroma of wines made from Cabernet Sauvignon grapes, as confirmed by several researchers [36, 46].

\subsection{Principal Component Analysis (PCA)}

Principal component analysis (PCA) was performed on the total volatile classes of each stage during development to correlate them with the different fertilizer treatments (Fig. 1). Principal component 1 (PC 1) explained $40.78 \%$ of the variance, while component 2 (PC 2) explained $24.34 \%$, representing $65.12 \%$ of the total variance. PC 1 was correlated with carbonyls (pre-veraison), alcohols (pre-veraison), terpenes (pre-veraison), esters (pre-veraison), terpenes (veraison), and alcohols (veraison) on the positive side and negatively correlated with carbonyls (maturity), terpenes (maturity), and esters (maturity). Carbonyls (pre-veraison), alcohols (pre-veraison), terpenes (pre-veraison), and esters (pre-veraison) were all strongly correlated to control (CK) samples but not so associated with terpenes (veraison) and alcohols (veraison). On the other hand, PC 2 positively correlated with alcohols (veraison) and esters (veraison) while correlating with carbonyls (veraison) negatively. Carbonyls (maturity), terpenes (maturity), and esters (maturity) were strongly connected to samples treated with OF on the negative side of PC 1, indicating that samples treated with $\mathrm{OF}$ are rich in carbonyls, terpenes, and esters during maturity but low compared to control during pre-veraison. $\mathrm{CF}$ and $1 / 2(\mathrm{O}+\mathrm{C})$ treated samples were closely associated with alcohols (veraison) and esters (veraison) on the positive side of $\mathrm{PC} 2$, while $\mathrm{O}+\mathrm{C}$ was correlating strongly with carbonyls (veraison) on the negative side. However, SC treatment was correlated inversely with alcohols (veraison) and esters (veraison). The results showed that different fertilizer applications had a distinct effect on grape volatile compounds accumulation during development. Coletta et al. [6] reported similar 


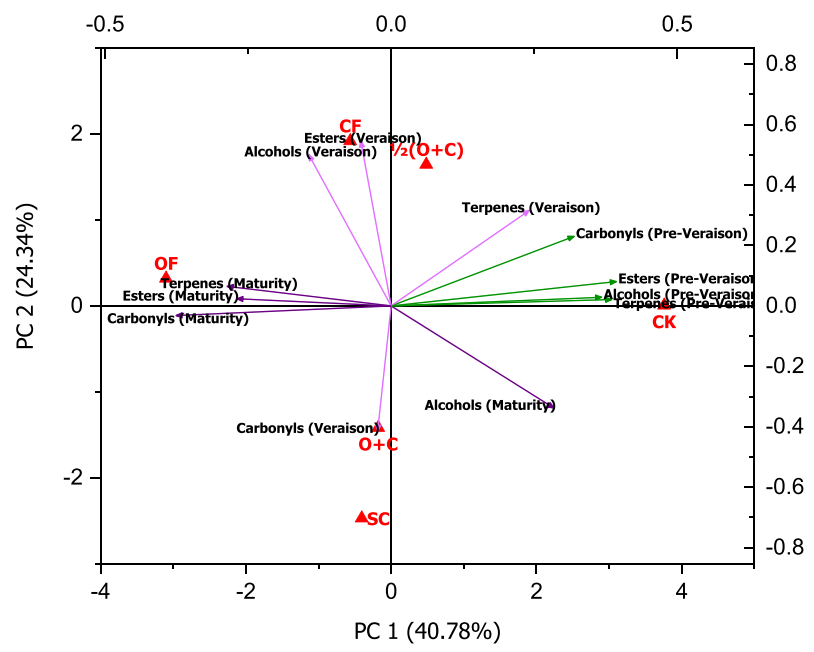

Fig. 1. Principal component analysis (PCA) was performed with grape volatile compound classes in Cabernet Sauvignon samples from untreated $(\mathrm{CK})$ and treated grapevines with different fertilization applications during development. CK, samples without any treatment; $\mathrm{CF}$, samples treated with chemical fertilizer; $\mathrm{OF}$, samples treated with organic fertilizer; $\mathrm{O}+\mathrm{C}$, samples treated with $50 \% \mathrm{CF}$ and $50 \% \mathrm{OF}$; $1 / 2(\mathrm{O}+\mathrm{C})$, samples treated with $25 \% \mathrm{CF}$ and $25 \% \mathrm{OF}$; SC, samples treated with soil conditioner.

observations where two different soil managements and training systems influenced the aroma composition of Negroamaro wines in the Puglia region of Southern Italy. Moreover, the results depict that samples treated with OF are more likely to increase esters and terpenes accumulations during maturity, which are the most aromatic volatile classes found in grapes [10].

\section{Conclusion}

The results showed that different fertilizer applications to Cabernet Sauvignon vines had significant influences on both chemical properties and the volatile components of the grapes. The type of fertilizer applied was the leading factor influencing the aroma profile of Cabernet Sauvignon grapes. The ratio of chemical and organic fertilizer combination was another factor that significantly affected the grapes. Samples treated with OF accumulated higher concentrations of carbonyls, terpenes, and esters during the maturity stage than other treated samples. Similarly, the accumulation and concentration of volatiles during veraison were higher for $\mathrm{CF}$ and $1 / 2(\mathrm{O}+\mathrm{C})$ samples than the others, except for $\mathrm{SC}$, which had a higher level of carbonyls during veraison. In addition, samples treated with SC accumulated more carbonyls and alcohols during the pre-veraison and veraison stages than those treated with $\mathrm{O}+\mathrm{C}$. The volatile concentrations of each chemical class throughout development show that the accumulation of volatile compounds depends on enzyme activity and specificity more than substrate concentration. Future studies on enzyme activity and gene expression will be required to fully comprehend the influence of these fertilizer treatments on the volatile compounds of Cabernet Sauvignon grapes. Also, the impact of these treatments on the phenolic components is essential since phenolic compounds can influence the sensorial qualities of wines.

\section{Acknowledgments}

The authors thank Wang Rui from Ningxia Agricultural University (Ningxia, China) for providing grape samples. 


\section{Funding}

This research was funded by the National Natural Science Foundation of China (32060514) and the National Key Research and Development Project of China (Grant No. 2019YFD1002500).

\section{Conflict of interest}

The authors have no conflict of interest to report.

\section{Supplementary material}

The supplementary material is available in the electronic version of this article: https://dx.doi.org/10.3233/JBR211517.

\section{References}

[1] Wu Y, Zhang W, Song S, Xu W, Zhang C, Ma C, et al. Evolution of volatile compounds during the development of Muscat grape 'Shine Muscat' (Vitis labrusca $\times$ V. vinifera). Food Chem [Internet]. 2020;309:125778. Available from: https://doi.org/10.1016/j.foodchem.2019.125778

[2] Jiang B, Zhang Z. Volatile compounds of young wines from cabernet sauvignon, cabernet gernischet, and chardonnay varieties grown in the loess plateau region of China. Molecules. 2010;15:9184-96.

[3] Alem H, Rigou P, Schneider R, Ojeda H, Torregrosa L. Impact of agronomic practices on grape aroma composition: a review. J Sci Food Agric. 2019;99(3):975-85.

[4] Jackson RS. Vineyard practice. Wine Science. 2020. pp. 151-330.

[5] Bell SJ, Henschke PA. Implications of nitrogen nutrition for grapes, fermentation, and wine. Aust J Grape Wine Res. 2005; 11(3):242-95.

[6] Coletta A, Toci AT, Pati S, Ferrara G, Grieco F, Tufariello M, et al. Effect of soil management and training system on negroamaro wine aroma. Foods. 2021;10(2):1-15.

[7] Zhang C, Jia H, Zeng J, Perraiz T, Xie Z, Zhu X, et al. Fertilization of Grapevine Based on Gene Expression. Plant Genome. 2016;9(3):plantgenome2015.09.0083.

[8] Lombardo VA, Osorio S, Borsani J, Lauxmann, M.A. Bustamante CA, Budde CO, Andreo CS, et al. Metabolic profiling during peach fruit development and ripening reveals the metabolic networks that underpin each developmental stage. Plant Physiol. 2011;157(4):1696-710.

[9] El-Badawy H. Implication of Using Potassium and Magnesium Fertilization to Improve Growth, Yield, and Quality of Crimson Seedless Grapes (Vitis vinifera L.). J Plant Prod. 2019;10(2):133-41.

[10] Gutiérrez-Gamboa G, Garde-Cerdán T, Carrasco-Quiroz M, Martínez-Gil AM, Moreno-Simunovic Y. Improvement of wine volatile composition through foliar nitrogen applications to 'Cabernet Sauvignon' grapevines in a warm climate. Chil J Agric Res. 2018;78(2):216-27.

[11] Helwi P, Habran A, Guillaumie S, Thibon C, Hilbert G, Renault C, et al. Can wine aromas be manipulated by nitrogen applications in the vineyard? In: International Conference WAC Proceedings of the International Conference. 2017.

[12] Lasa B, Menendez S, Sagastizabal K, Muro J, Idoia PMA. Foliar application of urea to "'Sauvignon Blanc"” and “"Merlot”" vines : doses and time of application. 2012;73-81.

[13] Garde-Cerdán T, Santamaría P, Rubio-Bretón P, González-Arenzana L, López-Alfaro I, López R. Foliar application of proline, phenylalanine, and urea to Tempranillo vines: Effect on grape volatile composition and comparison with the use of commercial nitrogen fertilizers. LWT - Food Sci Technol [Internet]. 2015;60(2):684-9. Available from: http://dx.doi.org/10.1016/j.lwt.2014.10.028

[14] Tang K, Tian X, Ma Y, Sun Y, Qi X, Miu C, et al. Aroma characteristics of Cabernet Sauvignon wines from Loess Plateau in China by QDA®, Napping ${ }^{\circledR}$ and GC-O analysis. Eur Food Res Technol [Internet]. 2020;246(4):821-32. Available from: https://doi.org/10.1007/s00217-020-03448-5

[15] Xu XQ, Liu B, Zhu BQ, Lan Y Bin, Gao Y, Wang D, et al. Differences in volatile profiles of Cabernet Sauvignon grapes grown in two distinct regions of China and their responses to weather conditions. Plant Physiol Biochem [Internet]. 2015;89:123-33. Available from: http://dx.doi.org/10.1016/j.plaphy.2015.02.020 
[16] Lei Y, Xie S, Guan X, Song C, Zhang Z, Meng J. Methoxypyrazines biosynthesis and metabolism in grape: A review. Food Chem [Internet]. 2018;245(November):1141-7. Available from: https://doi.org/10.1016/j.foodchem.2017.11.056

[17] Leeuwen C. van, Darriet P. The Impact of Climate Change on Viticulture and Wine Quality. Am Assoc of Wine Econ. 2016;11(1):15067.

[18] Gattullo CE, Mezzapesa GN, Stellacci AM, Ferrara G, Occhiogrosso G, Petrelli G, et al. Cover crop for a sustainable viticulture: Effects on soil properties and table grape production. Agronomy. 2020;10(9).

[19] Garde-Cerdán T, Lorenzo C, Lara JF, Prado F, Ancín-Azplicueta, Carmen Salinas MR. Study of the Evolution of Nitrogen Compounds during Grape Ripening. Application to Differentiate. J Agric Food Chem. 2009;57:2410-9.

[20] Canoura C, Kelly MT, Ojeda H. Effect of irrigation and timing and type of nitrogen application on the biochemical composition of Vitis vinifera L. cv. Chardonnay and Syrah grape berries. Food Chem. 2018;241:171-81.

[21] Bruwer FA, du Toit W, Buica A. Nitrogen and sulphur foliar fertilisation. South African J Enol Vitic. 2019;40(2):1-16.

[22] Yuan F, Qian MC. Development of C13-norisoprenoids, carotenoids, and other volatile compounds in Vitis vinifera L. cv. Pinot noir grapes. Food Chem. 2016;192(541):633-41.

[23] Liu M. Da, Wang QN, Wei JL, Zhang JT, Liu WE, Wang YJ. Effects of combined application of vermicompost and chemical fertilizer on yield and quality of Lactuca sativa. Chinese J Ecol. 2019;38(6):1760-6.

[24] Wang R, Ma L, Li L, Gu X, Sun Q. Influence of different fertilization treatments on soil fertility and wine grape quality. North Hortic. 2017; Chinese with English abstract.

[25] Torrea D, Varela C, Ugliano M, Ancin-Azpilicueta C, Leigh Francis I, Henschke PA. Comparison of inorganic and organic nitrogen supplementation of grape juice - Effect on volatile composition and aroma profile of a Chardonnay wine fermented with Saccharomyces cerevisiae yeast. Food Chem [Internet]. 2011;127(3):1072-83. Available from: http://dx.doi.org/10.1016/j.foodchem.2011.01.092

[26] González-Barreiro C, Rial-Otero R, Cancho-Grande B, Simal-Gándara J. Wine Aroma Compounds in Grapes: A Critical Review. Crit Rev Food Sci Nutr. 2015;55(2):202-18.

[27] OIV. International Organisation of Vine and Wine (OIV). Compendium of International Methods of Wine and Must Analysis. 2016; Volume 1.

[28] Ju YL, Xu GQ, Yue XF, Zhao XF, Tu T., Zhang JX, et al. Effects of regulated deficit irrigation on amino acid profiles and their derived volatile compounds in Cabernet Sauvignon (Vitis vinifera L.) grapes and wines. Molecules. 2018;23:1-13.

[29] Antalick G, Šuklje K, Blackman JW, Meeks C, Deloire A, Schmidtke LM. Influence of grape composition on red wine ester profile: Comparison between Cabernet Sauvignon and Shiraz cultivars from Australian warm climate. J Agric Food Chem. 2015;63(18):466472 .

[30] Deluc LG, Quilici DR, Decendit A, Grimplet J, Wheatley MD, Schlauch KA, et al. Water deficit alters differentially metabolic pathways affecting important flavor and quality traits in grape berries of Cabernet Sauvignon and Chardonnay. BMC Genomics. 2009;10:1-33.

[31] Soubeyrand E, Basteau C, Hilbert G, Van Leeuwen C, Delrot S, Gomès E. Nitrogen supply affects anthocyanin biosynthetic and regulatory genes in grapevine cv. Cabernet-Sauvignon berries. Phytochemistry [Internet]. 2014;103(July 2014):38-49. Available from: http://dx.doi.org/10.1016/j.phytochem.2014.03.024

[32] van Schalkwyk H, Archer E. Optimum Ripeness in Wine Grapes. WineLand [Internet]. 2000; Available from: https://www.wineland.co.za/optimum-ripeness-in-wine-grapes/

[33] Yue TX, Chi M, Song CZ, Liu MY, Meng JF, Zhang ZW, et al. Aroma characterization of cabernet sauvignon wine from the plateau of Yunnan (China) with different altitudes using SPME-GC/MS. Int J Food Prop [Internet]. 2015;18(7):1584-96. Available from: http://dx.doi.org/10.1080/10942912.2014.923442

[34] Ferrara G, Mazzeo A. Cover cropped and tilled table grape vineyard: Data on leaves and stems. Data Br [Internet]. 2021;35:106861. Available from: https://doi.org/10.1016/j.dib.2021.106861

[35] Canuti V, Conversano M, Calzi ML, Heymann H, Matthews MA, Ebeler SE. Headspace solid-phase microextraction-gas chromatography-mass spectrometry for profiling free volatile compounds in Cabernet Sauvignon grapes and wines. J Chromatogr A. 2009; 1216(15):3012-22.

[36] Kalua CM, Boss PK. Evolution of volatile compounds during the development of cabernet sauvignon grapes (Vitis vinifera L.). J Agric Food Chem. 2009;57(9):3818-30.

[37] El Hadi MAM, Zhang FJ, Wu FF, Zhou CH, Tao J. Advances in fruit aroma volatile research. Molecules. 2013;18(7):8200-29.

[38] Pasković I, Soldo B, Goreta Ban S, Radić T, Lukić M, Urlić B, et al. Fruit quality and volatile compound composition of processing tomato as affected by fertilisation practices and arbuscular mycorrhizal fungi application. Food Chem. 2021;359(July 2020).

[39] Yuan F. Grape and Wine Aroma Influenced by Vine Nutrient Status, Vigor and Crop Levels in Oregon Pinot Noir. Vol. 01, Journal of Chemical Information and Modeling. Oregon State University; 2016.

[40] Cheng X, Liang Y, Zhang A, Wang P, He S, Zhang K, et al. Using foliar nitrogen application during veraison to improve the flavor components of grape and wine. J Sci Food Agric. 2021;101(4):1288-300. 
[41] Garde-Cerdán T, Gutiérrez-Gamboa G, López R, Rubio-Bretón P, Pérez-Álvarez EP. Influence of foliar application of phenylalanine and urea at two doses to vineyards on grape volatile composition and amino acids content. Vitis - J Grapevine Res. 2018;57(4):137-41.

[42] Yuan F, Paul Schreiner R, Qian MC. Soil nitrogen, phosphorus, and potassium alter $\beta$-damascenone and other volatiles in pinot noir berries. Am J Enol Vitic. 2018;69(2):157-66.

[43] García E, Chacón JL, Martínez J, Izquierdo PM. Changes in volatile compounds during ripening in grapes of Airén, Macabeo and Chardonnay white varieties grown in La Mancha region (Spain). Food Sci Technol Int. 2003;9(1):33-41.

[44] Osorio S, Muñoz C, Valpuesta V. Physiology and Biochemistry of Fruit Flavors. Handb Fruit Veg Flavors. 2010;25-43.

[45] Sánchez Palomo E, Díaz-Maroto Hidalgo MC, González-Viñas MÁ, Pérez-Coello MS. Aroma enhancement in wines from different grape varieties using exogenous glycosidases. Food Chem. 2005;92(4):627-35.

[46] Gutiérrez-Gamboa G, Garde-Cerdán T, Rubio-Bretón P, Pérez-Álvarez EP. Seaweed foliar applications at two dosages to Tempranillo blanco (Vitis vinifera L.) grapevines in two seasons: Effects on grape and wine volatile composition. Food Res Int [Internet]. 2020;130:108918. Available from: https://doi.org/10.1016/j.foodres.2019.108918

[47] Yang Y, Li M, Zhang Z, Peng B. Correlation analysis of key enzyme activities and aroma compounds during fermentation of simulated juice system with Saccharomyces cerevisiae. Lwt [Internet]. 2019;108(December 2018):214-20. Available from: https://doi.org/10.1016/j.lwt.2019.03.072

[48] Aragüez I, Valpuesta Fernández V. Metabolic engineering of aroma components in fruits. Biotechnol J. 2013;8(10):1144-58.

[49] Kalua CM, Boss PK. Comparison of major volatile compounds from Riesling and Cabernet Sauvignon grapes (Vitis vinifera L.) from fruitset to harvest. Aust J Grape Wine Res. 2010;16(2):337-48.

[50] Vilanova M, Fandiño M, Frutos-Puerto S, Cancela JJ. Assessment fertigation effects on the chemical composition of Vitis vinifera L. cv. Albariño. Food Chem [Internet]. 2019;278(September 2018):636-43. Available from: https://doi.org/10.1016/j.foodchem.2018.11.105

[51] Beekwilder J, Alvarez-Huerta M, Neef E, Verstappen FWA, Bouwmeester HJ, Aharoni A. Functional Characterization of Enzymes Forming Volatile Esters from Strawberry and Banana. Plant Physiol [Internet]. 2004;135:1865-78. Available from: http://www.cultura.gov.br/documents/10883/1171222/cadernoPNLL_2014ab.pdf/df8f8f20-d613-49aa-94f5-edebf1a7a660

[52] Defilippi BG, Manríquez D, Luengwilai K, González-Agüero M. Chapter 1 Aroma Volatiles. Biosynthesis and Mechanisms of Modulation During Fruit Ripening. Adv Bot Res. 2009;50(08):1-37.

[53] Waterhouse AL, Sacks GL, Jeffery DW. Understanding Wine Chemistry. Understanding Wine Chemistry. 2016.

[54] Swiegers JH, Bartowsky EJ, Henschke PA, Pretorius IS. Yeast and bacterial modulation of wine aroma and flavour. Aust J Grape Wine Res. 2005;11(2):139-73.

[55] Sánchez-Gómez R, Torregrosa L, Zalacain A, Ojeda H, Bouckenooghe V, Schneider R, et al. Behavior of glycosylated aroma precursors in Microvine fruits after guaiacol foliar application. Sci Hortic (Amsterdam) [Internet]. 2019;246(September):e1-8. Available from: https://doi.org/10.1016/j.scienta.2018.11.068

[56] Zhang P, Fuentes S, Siebert T, Krstic M, Herderich M, Barlow EWR, et al. Terpene evolution during the development of Vitis vinifera L. cv. Shiraz grapes. Food Chem. 2016;204:463-74.

[57] Pott DM, Osorio S, Vallarino JG. From central to specialized metabolism: An overview of some secondary compounds derived from the primary metabolism for their role in conferring nutritional and organoleptic characteristics to fruit. Front Plant Sci. 2019;10(June). 